\title{
Antisense transcription licenses nascent transcripts to mediate transcriptional gene silencing
}

\author{
Yunkun Dang, ${ }^{1,4}$ Jiasen Cheng, ${ }^{2,4}$ Xianyun Sun, ${ }^{3}$ Zhipeng Zhou, ${ }^{1}$ and Yi Liu ${ }^{1}$ \\ ${ }^{1}$ Department of Physiology, University of Texas Southwestern Medical Center, Dallas, Texas 75390, USA; ${ }^{2}$ State Key Laboratory \\ of Agricultural Microbiology, College of Plant Science and Technology, Huazhong Agricultural University, Wuhan, Hubei 430070, \\ China; ${ }^{3}$ State Key Laboratory of Mycology, Institute of Microbiology, Chinese Academy of Sciences, ZhongGuanCun, Beijing \\ 100080, China
}

In eukaryotes, antisense transcription can regulate sense transcription by induction of epigenetic modifications. We showed previously that antisense transcription triggers Dicer-independent siRNA (disiRNA) production and disiRNA locus DNA methylation (DLDM) in Neurospora crassa. Here we show that the conserved exonuclease ERI-1 (enhanced RNAi-1) is a critical component in this process. Antisense transcription and ERI-1 binding to target RNAs are necessary and sufficient to trigger DLDM. Convergent transcription causes stalling of RNA polymerase II during transcription, which permits ERI-1 to bind nascent RNAs in the nucleus and recruit a histone methyltransferase complex that catalyzes chromatin modifications. Furthermore, we show that, in the cytoplasm, ERI-1 targets hundreds of transcripts from loci without antisense transcription to regulate RNA stability. Together, our results demonstrate a critical role for transcription kinetics in long noncoding RNA-mediated epigenetic modifications and identify ERI-1 as an important regulator of cotranscriptional gene silencing and post-transcriptional RNA metabolism.

[Keywords: DNA methylation; Neurospora; antisense transcription; gene silencing; small RNA]

Supplemental material is available for this article.

Received June 17, 2016; revised version accepted October 21, 2016.

Natural antisense RNAs (NATs), a major class of long noncoding RNAs that overlap with and are transcribed independently from sense RNAs, are widespread in the eukaryotic genomes (Kampa 2004; Katayama 2005; David 2006; Donaldson and Saville 2012; Magistri et al. 2012). NATs are involved in diverse physiological processes such as the circadian clock, $\mathrm{X}$ chromosome inactivation, imprinting, and cell fate determination in fungi, plants, and animals (Kramer et al. 2003; Camblong et al. 2007; Osato et al. 2007; Nagano et al. 2008; Halic and Moazed 2010; Deuve and Avner 2011; Xue et al. 2014). Although NATs can potentially hybridize with sense RNAs to form dsRNAs and siRNAs, most known functions of antisense transcription result from its roles in epigenetic silencing. In cis, the transcription of NATs can lead to various epigenetic modifications, such as methylation of histone 3 (H3K9me3, H3K27me3, and H3K36me3) and DNA, which are repressive chromatin marks (Tufarelli et al. 2003; Navarro et al. 2006; Yu et al. 2008; Li et al. 2012; Xi et al. 2014). Although the mechanisms of these

\footnotetext{
${ }^{4}$ These authors contributed equally to this work.

Corresponding author: yi.liu@utsouthwestern.edu

Article published online ahead of print. Article and publication date are online at http://www.genesdev.org/cgi/doi/10.1101/gad.285791.116.
}

antisense actions are largely unknown, it has been proposed that NATs recruit histone/DNA modification enzymes (Sun et al. 2006; Zhao et al. 2008; Li et al. 2012). In addition, overlapping convergent transcription may cause RNA polymerase II (Pol II) stalling during elongation that may disrupt gene expression (Prescott and Proudfoot 2002; Osato et al. 2007; Hobson et al. 2012; Xue et al. 2014).

The filamentous fungus Neurospora crassa is a well-established model for study of epigenetic regulation of gene expression. Previous RNA profiling revealed 477 overlapping sense-antisense pairs in Neurospora (Arthanari et al. 2014). One of the best-characterized NATs is qrf, which is antisense to the circadian clock gene frequency (frq) (Kramer et al. 2003).frq transcription and qrf transcription mutually inhibit each other to form a double negative feedback loop that is required for circadian rhythmicity (Xue et al. 2014). Transcription of both genes results in transcriptional interference in the form of Pol II stalling

(C) 2016 Dang et al. This article is distributed exclusively by Cold Spring Harbor Laboratory Press for the first six months after the full-issue publication date (see http://genesdev.cshlp.org/site/misc/terms.xhtml). After six months, it is available under a Creative Commons License (Attribution-NonCommercial 4.0 International), as described at http:// creativecommons.org/licenses/by-nc/4.0/. 
and H3K36me3. In addition, H3K9me3 and DNA methylation were also found in the promoter region of $f_{q} q$ (Belden et al. 2011; Dang et al. 2013; Li et al. 2015).

In addition to canonical siRNAs and microRNAs, we previously discovered a unique type of small RNA (sRNA) called disiRNAs (Dicer-independent siRNAs) (Lee et al. 2010; Chang et al. 2012). Like siRNAs, disiRNAs map to both strands of the genome and are associated with the Argonaute protein QDE-2, but their biogenesis is independent of Dicer. RNA profiling revealed that most of the disiRNA loci produce overlapping antisense RNA (Lee et al. 2010). Recently, we discovered that disiRNA loci are associated with DNA methylation and H3K9me3 (Dang et al. 2013). Unlike the previously characterized DNA methylation events in repeat-induced point mutation regions in Neurospora (Galagan and Selker 2004), the disiRNA locus DNA methylation (DLDM) is dependent on transcription and is induced by antisense transcription (Dang et al. 2013). Interestingly, frq resides in one of the disiRNA loci (disiRNA-47), and H3K9me3 and DNA methylation in the promoter region of frq require qrf expression (Belden et al. 2011; Dang et al. 2013; Li et al. 2015).

We sought to determine how antisense transcription results in disiRNA production and histone and DNA methylation. In this study, we show that the conserved exonuclease ERI-1 (enhanced RNAi-1) is a critical component in DLDM triggered by antisense transcription and in disiRNA production. Our results show that antisense transcription allows ERI-1 to distinguish between nuclear and cytoplasmic RNA targets. Antisense transcription results in Pol II stalling during transcription, which allows ERI-1 to bind to RNA and recruit a histone methyltransferase complex to mediate histone methylation and DNA methylation in the nucleus. In addition, ERI-1 also regulates mRNA stability of cytoplasmic RNA targets. This study establishes how antisense RNA regulates chromatin structure by regulating transcription kinetics and identifies ERI-1 as a critical component of transcriptional gene silencing and post-transcriptional RNA degradation.

\section{Results}

ERI-1 is required for DLDM and SRNA production at disiRNA loci

We reasoned that components important for disiRNA production would be involved in DLDM. We examined the DNA methylation level at the disiRNA-47 locus in Neurospora knockout mutants from the Neurospora Knockout Consortium (Colot et al. 2006) in which genes likely to be involved in RNA metabolism were disrupted. One mutant, in which NCU06684 was disrupted, showed almost complete loss of DNA methylation at the disiRNA-47 locus. NCU06684 encodes a protein with an ERI-1-type DEDDh $3^{\prime}-5^{\prime}$ exonuclease domain and is the closest sequence homolog of the Caenorhabditis elegans and Schizosaccharomyces pombe ERI-1 protein (Kennedy et al. 2004; Iida et al. 2006). Thus, we named NCU06684 eri-1. In C. elegans,
ERI-1 was shown to be an exonuclease that suppresses exogenous double-stranded sRNA-mediated RNAi. It is also required for the production of the ERI-1 class of endogenous siRNAs (endo-siRNAs) in worms (Kennedy et al. 2004; Duchaine et al. 2006; Iida et al. 2006).

Genomic DNA from the eri-1 $1^{K O}$ and wild-type strains was digested with isoschizomers DpnII or BfuCI. Both of these endonucleases recognize GATC sites, but the latter is unable to digest sites modified with 5-methylcytosine $(5 \mathrm{mC})$. Southern blot analysis of the digested DNA samples revealed that the DLDM levels at the disiRNA-47 and disiRNA-29 loci were both dramatically decreased in the eri-1 ${ }^{K O}$ mutant compared with levels in the wildtype strain (Fig. 1A). In contrast, DNA methylation at the $\zeta-\eta$ region, which has canonical repeat-induced point mutation (RIP)-induced DNA methylation, was unaffected in the eri-1 ${ }^{K O}$ strain, indicating that ERI-1 specifically affects DLDM.

We next examined the disiRNA levels in the eri-1 ${ }^{K O}$ mutant by Northern analyses. As shown in Figure 1B, disiRNA levels from the disiRNA-47 and disiRNA-29 loci were dramatically decreased in the eri-1 ${ }^{K O}$ mutant compared with those in the wild-type strain. To further confirm our results, we performed sRNA sequencing (sRNA-seq) and methylated DNA immunoprecipitation (MeDIP) sequencing (MeDIP-seq). As expected, both disiRNA and DLDM levels were dramatically decreased at the disiRNA-47 and disiRNA-29 loci in the eri-1 ${ }^{K O} \mathrm{mu}-$ tant (Fig. 1C). Taken together, these data suggest that ERI-1 is required for disiRNA biogenesis and DLDM.

\section{DLDM can occur independently of the sRNA pathway}

We next examined the role of disiRNA in DLDM. We reasoned that if DLDM is caused by disiRNA produced via an sRNA-induced silencing pathway, then the disiRNA should also be able to act in trans to trigger DNA methylation at homologous DNA regions. To test this hypothesis, we created a strain in which a Pfrq-luc transgene (a luciferase gene driven by the $f r q$ promoter) was introduced at the his-3 locus in a wild-type strain (Fig. 2A). The Pfrq region of the transgene is homologous to the endogenous disiRNA-47 region, but the transgene lacks antisense transcription (Dang et al. 2013). As expected, robust DNA methylation from the transgene locus but not from a control locus (qa-2) was detected by the MeDIP assay (Fig. 2B), suggesting that the disiRNAs produced from endogenous disiRNA-47 loci mediate DNA methylation.

To exclude the possibility that the binding of transcription factor WHITE COLLAR complex (WCC) at the Pfrq region is responsible for the DNA methylation, we created another reporter construct targeted to the his-3 locus. In this construct (Pfrq-t.gfp.Pqa), the WCC-binding sites were replaced by a $g f p$ gene driven by a quinic acid (QA)inducible qa-2 promoter that is in the opposite orientation of Pfrq (Fig. 2C). This construct was introduced into wildtype, eri-1 ${ }^{K O}, f r q^{10}$ (a frq-null strain lacking the region producing disiRNA), and $q d e-2-n u l l\left(q d e-2^{R I P}\right)$ strains. As shown in Figure 2D, robust DNA methylation was 
A

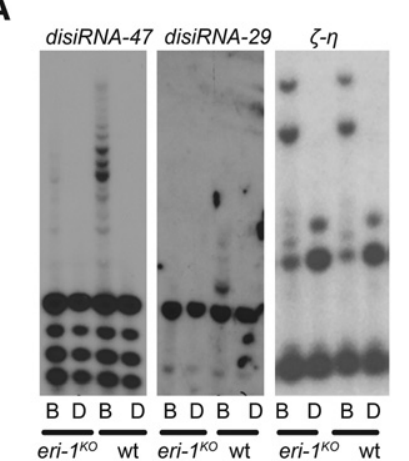

B

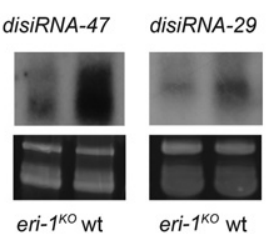

C
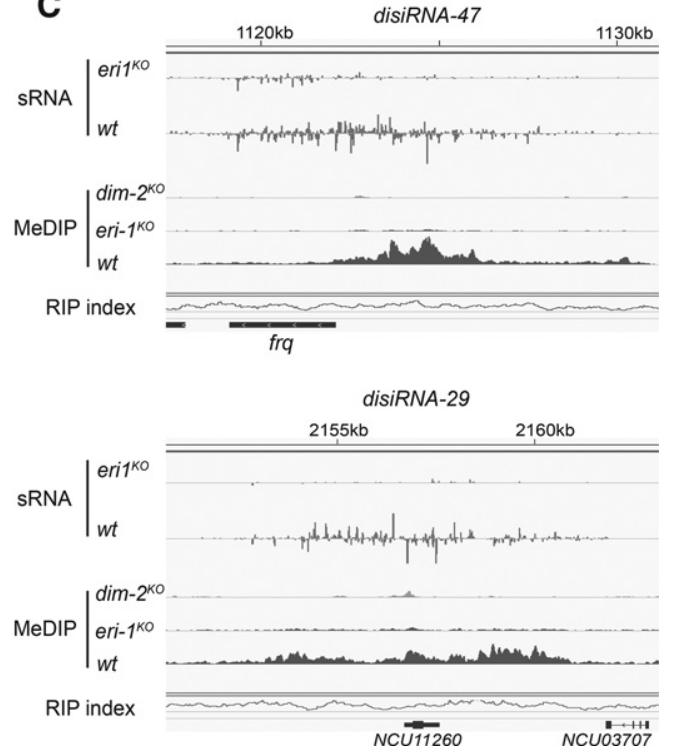

Figure 1. DLDM and disiRNA production are deficient in the eri-1 ${ }^{K O}$ strain. (A) Southern blot analysis results showing the methylation profiles of the disiRNA-47 and disiRNA-29 loci in the eri- $1^{K O}$ and wild-type strains. The $\zeta-\eta$ locus is a methylated DNA region due to RIP. Genomic DNA samples were digested with BfuCI (B) and DpnII (D) and probed with the corresponding probes. (B) Northern blot analysis of sRNA showing the level of disiRNAs from disiRNA-47 and disiRNA-29 loci in the indicated strains. The bottom panels show the loading of RNAs on denaturing gel stained with ethidium bromide. $(C)$ Comparison of levels of disiRNA and DNA methylation based on sRNA sequencing and MeDIP-seq (methylated DNA immunoprecipitation [MeDIP] combined with sequencing) results in the indicated strains. MeDIP-seq results of the $d i m-2^{K O}$ mutant served as nega-

tive controls. The horizontal line in the RIP index panel indicates the threshold $(<0.7)$ to trigger DNA methylation by RIP $($ Margolin et al. 1998).

also detected at the transgene locus in the wild-type background and was further increased upon induction of antisense transcription. Importantly, such DNA methylation was almost completely abolished in the $q d e-2^{R I P}$ and frq-null strains (Fig. 2D,E), indicating that DNA methylation at the transgene locus is triggered by disiRNAs produced from the endogenous disiRNA-47 locus through a QDE-2-dependent pathway. Furthermore, the transgene methylation was also nearly abolished in the eri-1 ${ }^{K O}$ strain, indicating that ERI-1 is essential in this pathway (Fig. 2E).

The results above indicate that disiRNA can mediate DNA methylation in a QDE-2- and RNA-dependent manner, suggesting that disiRNA guides QDE-2 to nascent RNA to mediate DNA methylation. Consistent with this conclusion, we had previously discovered disiRNA due to its association with QDE-2 (Lee et al. 2010). The complete dependence on QDE-2 for disiRNA action in trans prompted us to examine whether DLDM is dependent on QDE-2. Surprisingly, DLDM at the disiRNA-47 and disiRNA-29 loci was maintained in the $q d e-2^{R I P} \mathrm{mu}-$ tant and qde-2 ${ }^{R I P} ; s m s-2^{K O}$ double mutant $(s m s-2$ encodes for an argonaute protein specifically expressed during meiosis) compared with levels in the wild-type strain (Fig. 2G; Supplemental Fig. S1A). In addition, H3K9me3 chromatin immunoprecipitation (ChIP) analysis showed that $\mathrm{H} 3 \mathrm{~K} 9 \mathrm{me} 3$ was also maintained in the qde-2 mutant (Supplemental Fig. S1B). Furthermore, when an artificial construct that expresses convergent transcription and disiRNA (Dang et al. 2013) was introduced at the his-3 locus in the wild-type and $q d e-2^{R I P}$ strains, similar DLDM levels at the transgene locus were observed (Supplemental Fig. S1C). Taken together, these results indicate that disiRNA contributes to, but is not required for, DLDM and H3K9me3 at disiRNA loci.
ERI-1 is an exonuclease that specifically binds to a G-rich element at the $3^{\prime}$ ends of target $R N A$ s

ERI-1 homologs in mice and C. elegans contain putative RNA-binding SAP domains. Instead, the Neurospora ERI-1 has a putative RNA recognition motif (RRM). Compared with the wild-type strain, the eri-1 ${ }^{K O}$ mutant produces significantly fewer asexual spores but more aerial hyphae and has a "fluffy" appearance on slants with solid medium (Fig. 3A). The phenotype of the eri-1 ${ }^{K O}$ strain was rescued by an eri-1 transgene expressing Flag-tagged ERI-1 (eri-1 ${ }^{K O}$;Flag-ERI-1) but not by an ERI-1 transgene with the exonuclease catalytic residue mutated $\left(e r i-1^{K O} ;\right.$ FlagERI- $1^{\mathrm{CD}}$ ) or with the RRM deleted (eri- $1^{\mathrm{KO}}$;Flag-ERI$1^{\triangle \mathrm{RRM}}$ ). Similarly, the expression of wild-type ERI-1 but not the two mutated ERI-1 transgenes rescued DNA methylation defects of eri-1 ${ }^{K O}$ (Fig. 3B). These results indicate that the exonuclease and RNA-binding activities are required for ERI-1 function.

To further understand how ERI-1 functions, we focused on the disiRNA-47 locus, which includes the frq gene. frq encodes the well-studied central circadian clock protein FRQ (Dunlap 2006; Heintzen and Liu 2007). In addition to the sense $f_{r} q$ transcript, the locus also expresses an antisense transcript qrf that almost completely overlaps with frq (Fig. 3C; Kramer et al. 2003; Xue et al. 2014). Because the RRM domain of ERI-1 is required for its function in DLDM, we examined whether frq mRNA is a direct target of ERI-1 by RNA immunoprecipitation assays using a monoclonal Flag antibody. In the eri-1 ${ }^{\mathrm{KO}}$;FlagERI-1 strain, little binding of ER1 to the central region of the frq transcript was observed, but we observed a strong binding preference to both $5^{\prime}$ and $3^{\prime}$ ends of RNA from the frq locus (Fig. 3C). In contrast, such binding was completely absent in the eri-1 ${ }^{K O} ;$ Flag-ERI-1 ${ }^{\triangle \mathrm{RRM}}$ strain. 
A
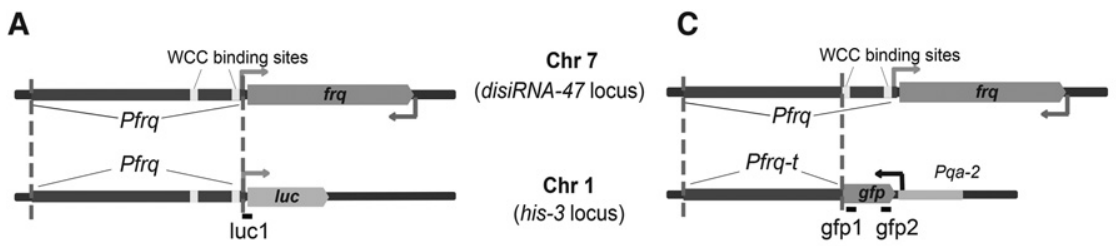

B

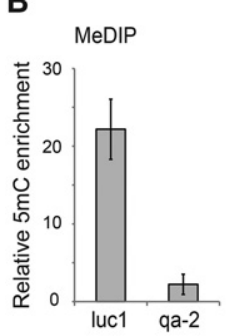

D

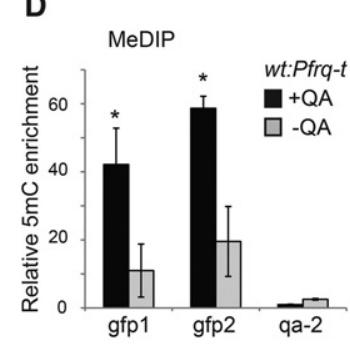

E

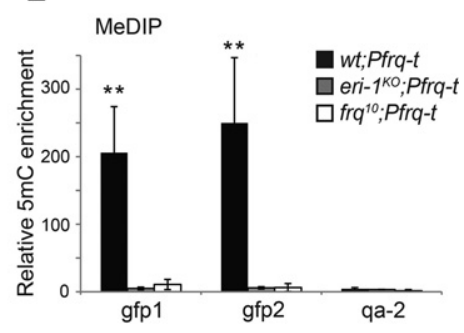

$\mathbf{F}$

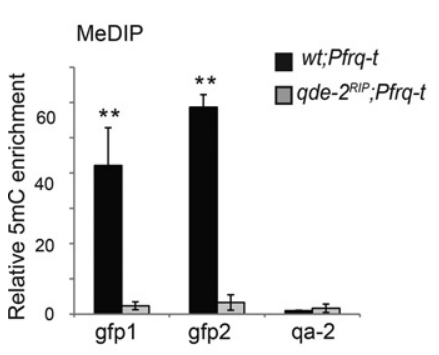

G

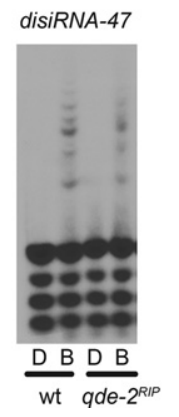

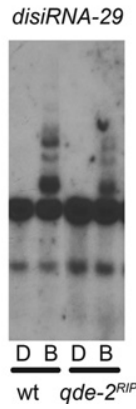

$\zeta-\eta$

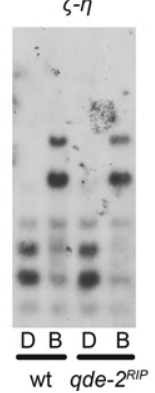

Figure 2. disiRNAs contribute to but are not required for DLDM. (A) Schematic view of the disiRNA-47 locus (on chromosome 7) and the luciferase transgene (Pfrq. luc) at the his-3 locus (on chromosome 1). The region between the two dotted lines indicates the identical sequence region shared between the endogenous frq promoter and the transgene. The short black bars (labeled as luc1) indicate the approximate locations of primer sets used to detect DNA methylation by MeDIP. (B) MeDIP results of the wild-type strain harboring the luciferase reporter construct (wt:Pfrq.luc). The qa-2 locus was used as a negative control. Results were obtained from three independent experiments. Values are mean $\pm \mathrm{SD}$. (C) Schematic view of the disiRNA-47 locus and the Pfrq-t.gfp.Pqa transgene at the his-3 locus. The region between the two dotted lines indicates the identical sequence region shared between the endogenous $f r q$ promoter and the transgene. The short black bars indicate the approximate locations of primer sets used to detect DNA methylation by MeDIP. $(D-F)$ MeDIP results showing the relative DNA methylation levels of the $g f p$ transgene in the indicated strains. The qa-2 locus, which is located in endogenous qa- 2 gene but not on the Pqa-2, was used as a negative control. Results were obtained from three independent experiments. Unless indicated, cultures were grown in the presence of quinic acid. $\left.\left(^{*}\right) P<0.05 ;{ }^{* *}\right) P<0.01$, Student's t-test. $(G)$ Southern analyses of the disiRNA-47 and disiRNA-29 loci in the wild-type and $q d e-2^{R I P}$ strains. The $\zeta-\eta$ locus represents the DNA methylation associated with RIP. Genomic DNA samples were digested with BfuCI (B) and DpnII (D) and probed with the corresponding probes.

frq and $q r f$ mRNAs overlap almost completely, and the RT-qPCR assays here were not strand-specific. To understand how ERI-1 recognizes its target mRNAs, we performed high-throughput sequencing of RNA isolated by cross-linking immunoprecipitation (HITS-CLIP). FlagERI-1 was immunoprecipitated from the eri-1 ${ }^{\mathrm{KO}}$;FlagERI-1 strain. A wild-type strain that lacks Flag-ERI-1 expression was used as the negative control. The resulting sequencing reads were analyzed using the dCLIP software (Wang et al. 2014), and peaks of $>50$ nucleotides (nt) in width that were at least fivefold more intense than those in the wild-type control were identified. According to these criteria, 1805 peaks were identified, which were mapped to 1508 protein-coding genes (Supplemental Table S1). These data show that ERI-1 has a strong binding preference for the 3' untranslated region (UTR) (Fig. 3D, top panel). In contrast, such a preference was not observed in the control samples. Alignments of ERI-1-binding sites with transcriptional end sites (TESs) showed that the peaks of ERI-1 binding are located $200 \mathrm{nt}$ upstream of the $3^{\prime}$ transcript termini (Fig. 3D, bottom panel). Interestingly, the human ERI-1 homolog was also found previously to bind to the $3^{\prime}$ end of histone mRNA (Dominski et al. 2003).

To identify the ERI-1-binding motif on mRNA, we first used the PIPE-CLIP software (Chen et al. 2014) to pinpoint the cross-linked sites between RNA and ERI-1. Crosslinking sites could be identified by a nucleotide insertion or deletion during the reverse transcription step. As shown in Figure 3E (left), the consensus sequences flanking the ERI-1 cross-linked site show a strong preference for guanosine $(\mathrm{G})$ and adenosine $(\mathrm{A})$, with $\mathrm{G}$ as the dominant base. In addition, we also extracted the RNA sequences covered by ERI-1-binding peaks and identified consensus sequence patterns using the MEME suite (Bailey et al. 2009). Again, a G-rich motif was identified (Fig. $3 \mathrm{E}, \mathrm{middle})$. In contrast, the sequence motif generated from the control peaks in the control strain gave a very different sequence pattern (Fig. 3E, right). Together, these results suggest that ERI- 1 binds to the $3^{\prime}$ end of mRNAs with a G-rich motif.

We found that ERI-1 binds to a G-rich region near the $3^{\prime}$ ends of both frq and $q r f$ RNAs with major and minor binding sites (Fig. 3F). Our previous results showed that DNA methylation in the frq locus peaks in the frq promoter region, which is near the ERI-1-binding sites in the $3^{\prime}$ end of the qrf transcript (Dang et al. 2013). To experimentally determine the importance of the G-rich motif in mediating DLDM, we mutated either the major ERI-1-binding sites on $q r f$ or the sites on both $q r f$ and frq by introducing $\mathrm{G}$-to-C/T point mutations. The frq-rescuing constructs 
A

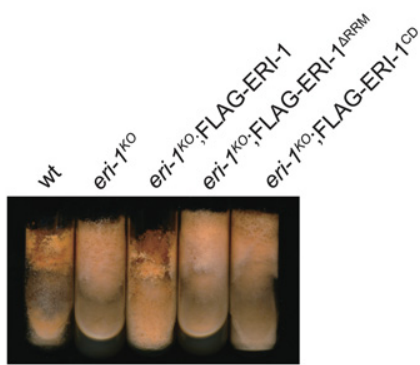

C
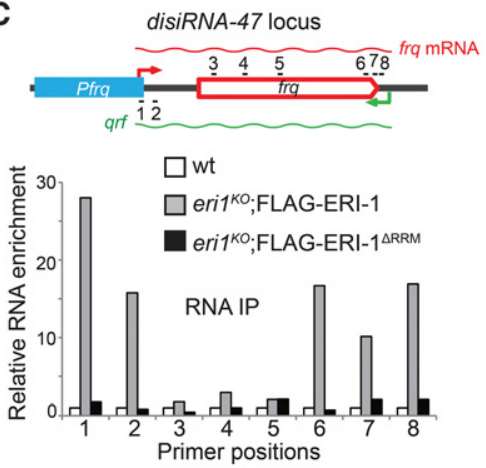

B

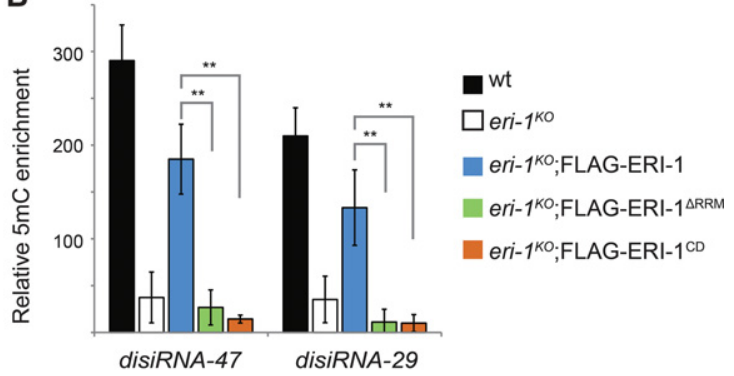

D

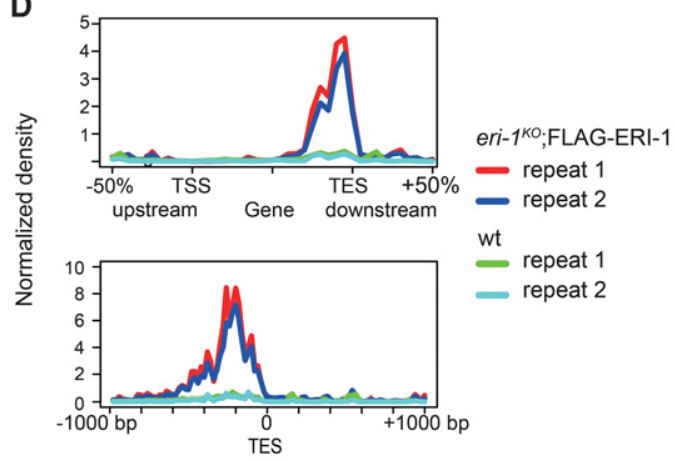

E

E $\quad$ ERI-1 binding sites

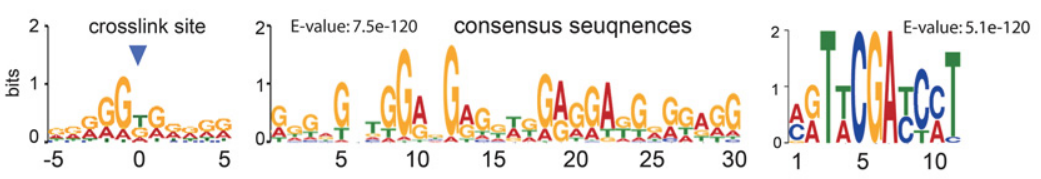

$\mathbf{F}$

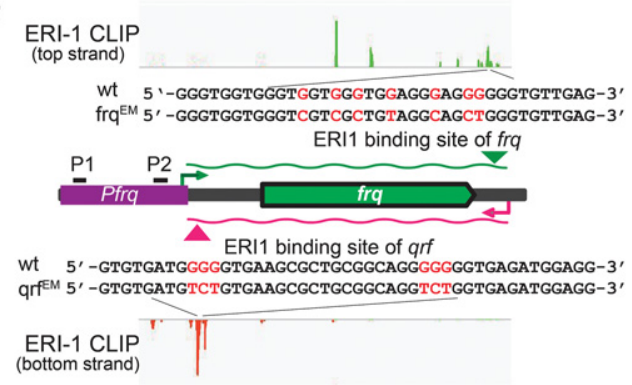

G

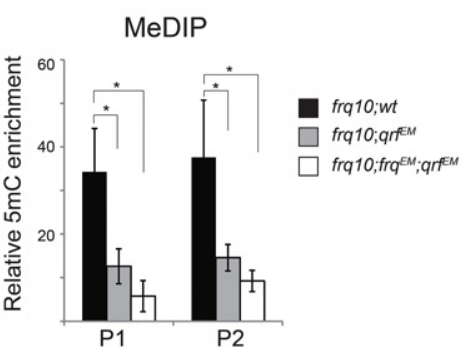

Figure 3. Characterization of ERI-1 and its RNA binding by HITS-CLIP (high-throughput sequencing of RNA isolated by cross-linking and immunoprecipitation). (A) The phenotype of the indicated strains grown in slants. (B) The DNA methylation levels of the indicated strains as determined by MeDIP. Three independent repeats were performed, and values represent mean \pm SD. $\left(^{* *}\right) P<0.01$, Student's $t$ test. $(C)$ The RNA immunoprecipitation assay of the indicated strains to determine the binding preference of ERI-1 and a mutant (ERI$1^{\triangle \mathrm{RRM}}$ ) on RNA from the disiRNA-47 locus. A schematic view of the disiRNA-47 locus and the frq and qrf genes is shown in the top panel. Numbers indicate the approximate positions of primer sets used in RNA immunoprecipitation. (D) Metagene plot of HITS-CLIP results showing ERI1-associated RNA fragment density in the eri-1 ${ }^{K O}$;Flag-ERI-1 strain and the wild-type strain (no-tag control). Each strain has two independent HITS-CLIP repeats. (TSS) Transcriptional start site; (TES) transcriptional end site. The top panel shows the relative position of ERI-1-binding preference by scaling target genes to the same length, whereas the bottom panel shows the absolute distance of ERI-1-binding position to the TES. (E) Consensus sequences of ERI1-targeted RNAs generated with the MEME suite. (Left) The sequences around the cross-linked sites (position 0) between target RNAs and ERI-1. (Middle) Consensus sequences from the alignments of ERI1associated RNA fragments. (Right) Consensus sequences from alignments of nonspecific peaks from control samples (wild-type strain). $(F)$ HITS-CLIP result showing the ERI-1-binding sites at the frq locus. The top and bottom strands are shown at the top and bottom, respectively. The red letters indicate the mutated nucleotides of the major ERI-1-binding sites on $f r q\left(\right.$ frq $\left.^{\mathrm{EM}}\right)$ and $q r f\left(\mathrm{qrf}^{\mathrm{EM}}\right)$ RNA as determined by HITS-CLIP. P1 and P2 are two primer sets used to determine DNA methylation. (G) MeDIP results of the relative levels of DNA methylation at the frq constructs at the his-3 locus with wild-type or mutated ERIl-binding sites on $q r f\left(f r q^{10} ; q r f^{E M}\right)$ or both $f r q$ and $q r f$ $\left(f r q^{10} ; f r q^{E M} ; q r f^{E M}\right)$, respectively. The results are based experiments using three independent transformants, and values represent mean \pm SD. $\left({ }^{*}\right) P<0.05$, Student's $t$-test. 
with these mutations ( $\mathrm{qrf}^{\mathrm{EM}}$ or both $\mathrm{frq}^{\mathrm{EM}}$ and $\mathrm{qrf}{ }^{\mathrm{EM}}$ ) and the wild-type frq construct were transformed into the frqnull strain $\left(f_{r q^{10}}\right)$. MeDIP analysis showed that the DLDM levels were markedly decreased in the $\mathrm{rrq}^{10}$; $\mathrm{qrf}^{\mathrm{EM}}$ and frq ${ }^{10}$; $\mathrm{frq}^{\mathrm{EM}} \mathrm{qrf}^{\mathrm{EM}}$ strains relative to the control (Fig. 3G), indicating that the ERI-1-binding sites are indeed critical for DLDM.

ERI-1 specifically targets nascent RNA from the disiRNA loci but not other RNAs in the nucleus

By HITS-CLIP, we identified 1500 mRNA transcripts targeted by ERI-1. These transcripts arise from most of the known disiRNA loci and many other non-disiRNA loci. To confirm the CLIP result, we performed RNA immunoprecipitation from whole-cell extracts for a group of these transcripts from disiRNA and non-disiRNA loci. As shown in Figure 4A, ERI-1 was specifically associated with these transcripts identified by HITS-CLIP as bound to ERI-1.

Even though ERI-1 recognizes hundreds of mRNAs, DLDM was not observed in non-disiRNA loci. Because DLDM is a nuclear event, we hypothesized that ERI-1 selectively targets the disiRNA loci for DLDM through differential binding in the nucleus versus the cytoplasm. Consistent with this notion, we observed ERI-1 in both the nucleus and cytoplasm (Supplemental Fig. S2). To test this hypothesis, we performed RNA immunoprecipitation from nuclear extracts. Strikingly, in nuclear extracts, the RNAs from the disiRNA loci exhibited robust binding to ERI-1, but no significant ERI-1 binding was detected for transcripts from the non-disiRNA loci (Fig. 4B). Therefore, in the nucleus, ERI-1 specifically targets transcripts from the disiRNA loci but not other target transcripts.

\section{Antisense transcription and ERI-1 binding of RNA are both necessary and sufficient to trigger DLDM}

Most disiRNA loci are known to produce antisense RNA, and previous studies have shown that convergent transcription leads to DLDM (Lee et al. 2010; Belden et al. 2011; Dang et al. 2013; Li et al. 2015). There are 477 genes in Neurospora that are known to produce antisense transcripts (Arthanari et al. 2014); therefore, antisense transcription alone is not sufficient to trigger DLDM. Based on the role of ERI-1 in DLDM, we hypothesized that DLDM might require both antisense transcription and ERI-1 binding. To test this hypothesis, we took advantage of the existing Neurospora knockout strains, which were created by replacing the ORF of the target gene with a gene that confers hygromycin resistance $(h p h)$ oriented opposite to the target gene (Colot et al. 2006). Therefore, the knockout locus has convergent transcription overlapping the $h p h$ gene (Fig. 4C). Thus, we predicted that DNA methylation would be found only in those knockout loci in which transcripts are targets of ERI-1. Based on our HITS-CLIP results, we examined 13 gene knockout strains. Among these genes (none produces an endogenous antisense tran- script), six have sense gene transcripts that were ERI-1bound, and seven were not. As expected, no DNA methylation was found in these 13 loci in a wild-type strain (Fig. 4D), confirming that ERI-1 binding alone is not sufficient to cause DNA methylation. As expected, robust DNA methylation was detected at the knockout loci in each of the six strains in which the transcripts are targets of ERI-1. In contrast, no methylation was found at the knockout loci of the seven strains in which transcripts are not ERI-1 targets.

To further confirm this conclusion, we focused on the $v v d$ locus. The sense $v v d$ transcripts have four ERI-1-binding sites (two major and two minor peaks) near the $3^{\prime}$ end, and very little DNA methylation of this locus is observed in the wild-type strain (Fig. 4E). As expected, the $v v d^{K O}$ locus was found to be associated with strong DNA methylation (Fig. 4F) as observed previously (Li et al. 2015). In addition, the DNA methylation at the $v v d^{K O}$ locus is independent of QDE-2 (Supplemental Fig. S3), further indicating the independence of DLDM from the RNAi pathway. We next created an eri-1 ${ }^{K O} ; v v d^{K O}$ double mutant. As expected, the DNA methylation level at the $v v d^{K O}$ locus was drastically reduced in the eri-1 ${ }^{K O} ; v v d^{K O}$ mutant compared with that of the $v v d^{K O}$ single mutant (Fig. 4F), indicating the critical role of ERI-1 in mediating DLDM induced by antisense transcription.

We then asked whether antisense transcription at the $v v d^{K O}$ locus resulted in ERI-1 binding to $h p h$ antisense transcripts in the nucleus. To address this question, we performed nuclear RNA immunoprecipitation in the eri$1^{K O}$;Flag-ERI-1 and $v v d^{K O}$;Flag-ERI-1 strains. Although only a background level of ERI-1 binding to $v v d$ RNA was detected in the eri-1 ${ }^{K O}$;Flag-ERI-1 strain, a strong binding between ERI- 1 and $v v d^{K O}$ RNAs (i.e., the $h p h$ antisense transcript) was seen in the $v v d^{K O}$; Flag-ERI-1 strain (Fig. 4G). As controls, we analyzed NCU00911 mRNA (in the disiRNA-6 locus) and NCU01229 transcripts (from a non-disiRNA locus); ERI-1 was found to be associated with NCU00911 mRNA but not with NCU01229 mRNA in both the eri-1 ${ }^{K O}$;Flag-ERI-1 and ${ }_{v V} d^{K O}$; Flag-ERI-1 strains. Taken together, these results indicate that antisense transcription and ERI-1 binding are necessary and sufficient to trigger DNA methylation. In addition, antisense transcription is required for ERI-1 recognition of RNAs with ERI-1-binding sites in the nucleus, which triggers DNA methylation.

\section{DLDM can be induced by slow down/stalling of transcription elongation/termination}

Convergent transcription has been proposed to result in Pol II stalling or collision during the transcription elongation process (Hobson et al. 2012). In Neurospora, we showed previously that antisense transcription leads to transcriptional interference and Pol II stalling at the frq (disiRNA-47) locus (Xue et al. 2014). Because of the requirement of convergent transcription in DLDM, we hypothesized that convergent transcription stalls or slows down Pol II, which provides time for ERI-1 to recognize nascent target RNAs attached to elongating Pol II. As a 
A

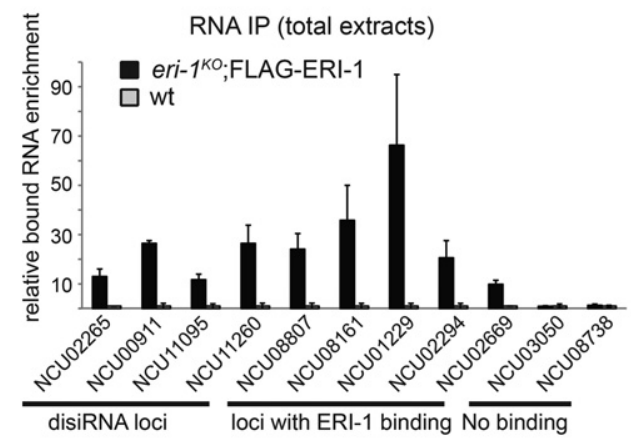

B

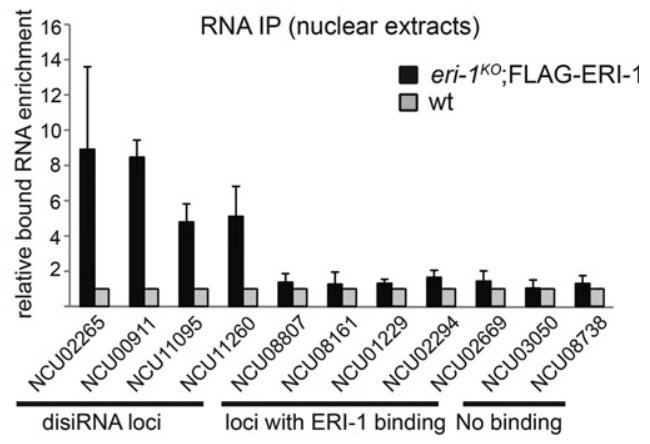

C
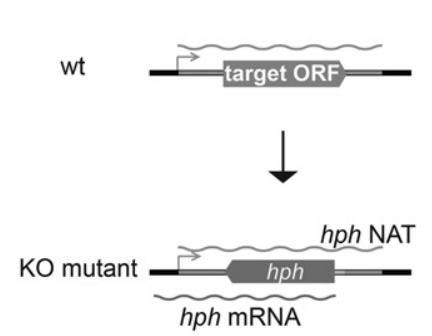

D

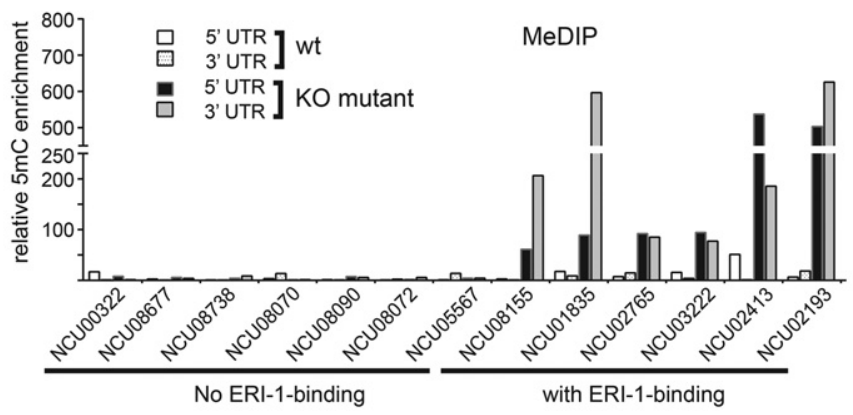

E

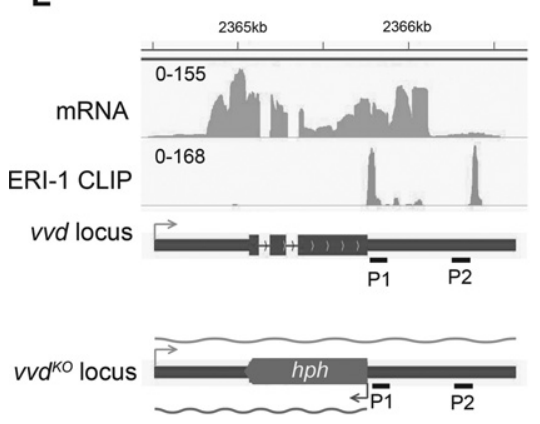

$\mathbf{F}$

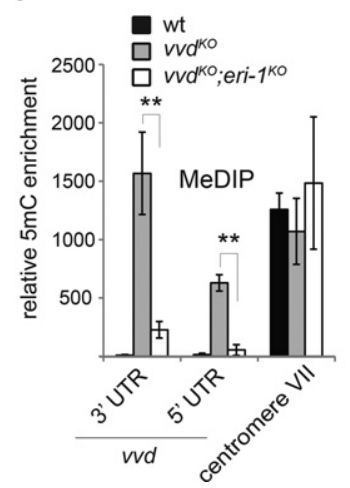

G

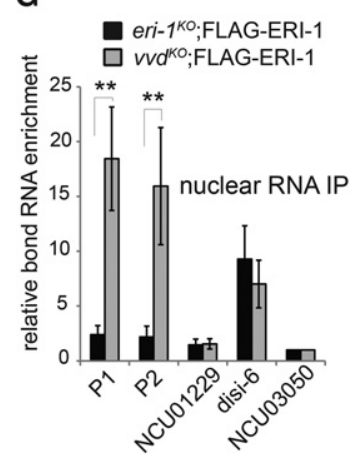

Figure 4. ERI-1 specifically targets nascent RNAs with antisense transcription in nucleus. $(A)$ RNA immunoprecipitation results of the indicated strains with total cell extracts. Primers for each gene were located at the $3^{\prime}$ ends of annotated gene transcripts, near the ERI-1binding peak as determined by HITS-CLIP. The results from wild type are set as 1 . For each strain, three independent repeats were carried out, and values represent mean \pm SD. $(B)$ RNA immunoprecipitation results of the indicated strains with nuclear extracts. $(C)$ Schematic view of the gene knockout locus in knockout mutants. The knockout locus has convergent transcription. $(D)$ MeDIP results showing relative DNA methylation levels from the knockout loci of 13 knockout mutants with or without targeting of ERI-1-binding transcripts. Primer sets were located at the $5^{\prime}$ and $3^{\prime}$ UTRs of the knockout gene. (E) mRNA-seq and HITS-CLIP results of the $v v d$ (NCU03967) locus. $\mathrm{P} 1$ and $\mathrm{P} 2$ indicate the positions of two primer pairs used for RNA immunoprecipitation. $(F)$ MeDIP results showing relative DNA methylation levels at the $5^{\prime}$ and $3^{\prime}$ UTRs of $v v d$ gene from the indicated strains. Three independent repeats were carried out, and values represent mean \pm SD. $\left({ }^{* *}\right) P<0.01$, Student's $t$-test. Centromere VII served as a positive control for DNA methylation. (G) RNA immunoprecipitation results with nuclear extracts from the eri-1 ${ }^{K O}{ }_{;}$Flag-ERIl strain and $v v d^{K O}{ }_{;}$Flag-ERI-1 strains. Three independent repeats were carried out, and values represent mean \pm SD. $\left(^{* *}\right) P<0.01$, Student's $t$-test.

result, ERI-1 can act to mediate methylation on nearby chromatin.

To test this hypothesis, we first examined that effect of 6-azauracil (6AU), an inhibitor of transcription elongation, on the level of DLDM. As shown in Figure 5A, the addition of 6AU resulted in a dose-dependent increase of DNA methylation levels at the disiRNA-29 and disiRNA-47 loci, suggesting that DLDM is triggered by retardation of transcription elongation. Moreover, the effect of 6AU is completely dependent on ERI-1 because the increase of 
Dang et al.

A

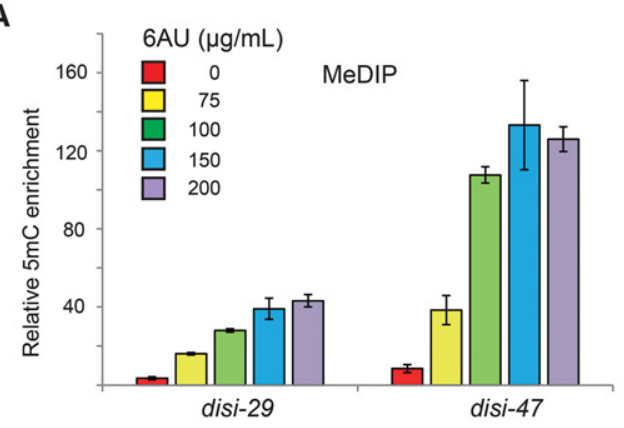

C
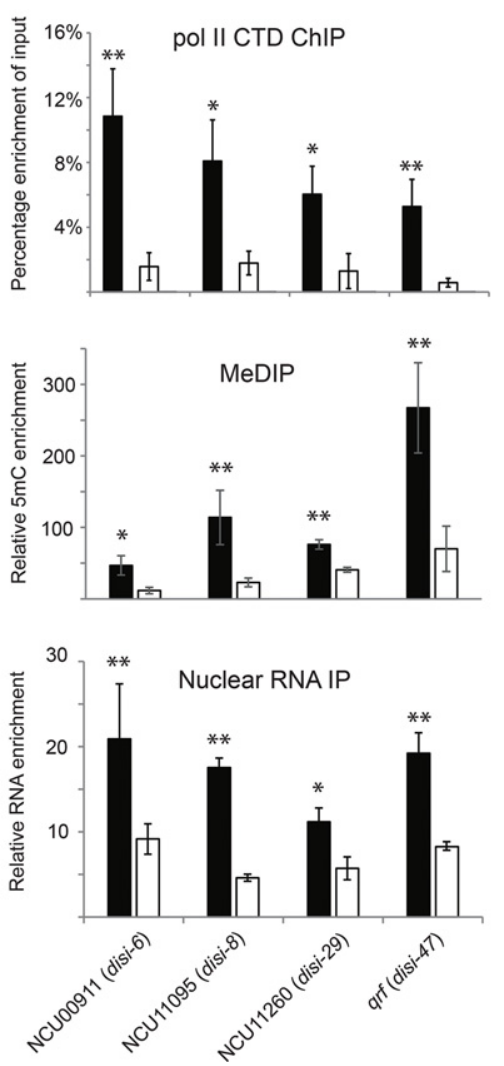

B

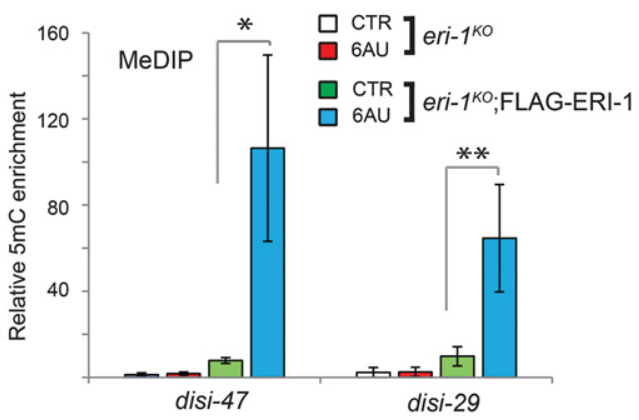

D
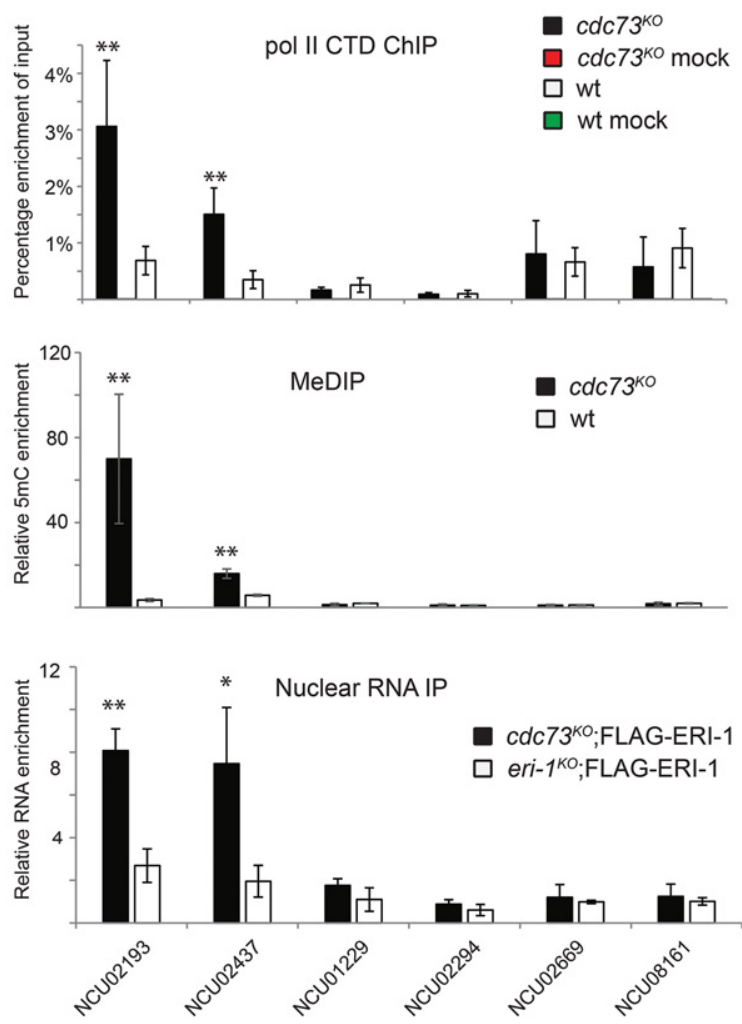

Figure 5. Pol II stalling and elevated DNA methylation levels at loci whose transcripts are targeted by ERI-1 in the $c d c 73^{K O}$ mutant. $(A)$ MeDIP assays showing the relative levels of $5 \mathrm{mC}$ at the disi-29 and disi-47 loci in wild-type cultures treated with the indicated 6AU concentrations. (B) MeDIP assays showing the relative levels of $5 \mathrm{mC}$ at the disi-29 and disi-47 loci in the eri-1 ${ }^{K O}$ and eri- ${ }^{K O}$;Flag-ERI-1 strains. (CTR) Control; (6AU) treated with $150 \mu \mathrm{g} / \mathrm{mL} 6 \mathrm{AU}$. Note that a different lot of the $5 \mathrm{mC}$ antibody was used from that used in the experiments in Figure 1. $(C, D)$ Pol II C-terminal domain $(\mathrm{CTD})$ ChIP assay (top) and MeDIP (middle) experiments showing the accumulation of Pol II and elevated DNA methylation at the indicated loci in the $c d c 73^{K O}$ and wild-type strains. The bottom panels show the results of the nuclear RNA immunoprecipitation assays between ERI-1 and RNA from the indicated loci in the $c d c 73^{K O}$ and wild-type strains. The Pol II CTD levels of the mutant were normalized to that of wild-type strain. For MeDIP experiment, the relative $5 \mathrm{mC}$ levels of tested regions were normalized to that of the am locus. Three independent repeats were carried out, and values represent mean \pm SD. $(*)$ $\left.P<0.05 ;{ }^{* *}\right) P<0.01$, Student's $t$-test.

DNA methylation by 6AU was completely abolished in the eri-1 ${ }^{K O}$ strain and was rescued by the expression of Flag-ERI-1 in the eri-1 ${ }^{K O}$ strain (Fig. 5B).

The RNA polymerase-associated factor 1 (Paf1) complex is a conserved five-subunit protein complex that regulates Pol II elongation and termination. The deletion of PaflC components such as Cdc73 impairs both elongation and termination efficiency (Rondón et al. 2004; Kim et al. 2010; Tomson and Arndt 2013). We performed ChIP assays to determine Pol II occupancy and DNA methylation levels at disiRNA loci in the Neurospora $c d c 73^{K O}$ strain. Consistent with the role of Paf1C in promoting Pol II elongation, ChIP assays using an antibody against the Pol II Cterminal domain (CTD) showed significant increases of 
Pol II occupancy at the regions corresponding to the $3^{\prime}$ ends of the ERI-1-targeted transcripts (Fig. 5C, top). To further confirm the transcription stalling at the $3^{\prime}$ ends of the target genes, we purified chromatin-associated nascent RNA from the $c d c 73^{K O}$ and control strains (Bhatt et al. 2012). As shown in Supplemental Figure S4A, the levels of chromatin-associated nascent RNA at these disiRNA loci were significantly elevated in the $c d c 73^{K O}$ mutant compared with the control strain. These results suggest that transcription at these loci is stalled in the $c d c 73^{K O}$ strain. As expected, methylation levels significantly higher than those in the wild-type strain were observed in the $c d c 73^{K O}$ strain at all disiRNA loci examined (Fig. 5C, middle).

If the stalling of transcription elongation/termination permits ERI-1 recognition of nascent transcripts with ERI-2-binding sites, we predicted that the loss of $c d c 73$ would lead to DNA methylation at some gene loci targeted by ERI-1 but lacking antisense transcription. We selected six non-disiRNA loci, all of which produced ERI-1-targeted mRNAs without antisense transcription or DNA methylation in a wild-type strain. The Pol II ChIP assays and chromatin-associated nascent RNA assay showed that Pol II occupancies and nascent RNA were significantly elevated in two of the six loci in the $c d c 73^{K O}$ strains compared with the wild-type strain (Fig. 5D, top; Supplemental Fig. S4B). The gene-specific effects of the Paf1C deficiency were expected to be due to the nonessential role of CDC73 and because Paf1C is known to preferentially affect the expression of a subset of genes (Penheiter et al. 2005). As expected, DNA methylation was detected at the two loci with elevated Pol II occupancy in the $c d c 73^{K O}$ strains but not in the wild-type strain (Fig. 5D, middle). In addition, nuclear RNA immunoprecipitation showed that ERI-1 bound to the transcripts from these same two loci in the $c d c 73^{K O}$ strain but not in the wild-type strains (Fig. 5D, bottom). Furthermore, 6AU treatment of wild-type cultures resulted ERI-1dependent DNA methylation at these two loci (Supplemental Fig. S5). Together, these results suggest that antisense transcription slows or stalls transcription elongation/termination to permit ERI-1 to bind to RNAs in the nucleus and mediate DNA methylation at nearby chromatin. Thus, regulation of transcription kinetics by antisense transcription plays an important role in mediating the epigenetic role of nascent RNA transcripts.

\section{ERI-1 associates with DDB1, a core component of the histone methyltransferase complex responsible for H3K $9 \mathrm{me} 3$}

Since ERI-1 lacks a protein domain known to mediate chromatin modification, we reasoned that it works in conjunction with other proteins to achieve histone/DNA methylation. To test this hypothesis, we immunoprecipitated Flag-ERI-1 from the ERI-1 ${ }^{K O}$;Flag-ERI-1 strain and used mass spectrometry to identify ERI-1-associated proteins. DDB1, a core component of the histone methyltransferase complex (DCDC) required for H3K9me3 and DNA methylation at the repeat-induced point-mutated loci (Le- wis et al. 2010; Zhao et al. 2010), was specifically enriched in the Flag immunoprecipitates from the eri-1 ${ }^{K O}$;Flag-ERI1 strain compared with the wild-type strain lacking FlagERI-1 expression. In Neurospora, H3K9me3 is known to recruit HP1, a heterochromatin-binding protein, and DIM-2, the DNA methyltransferase, which then lead to DNA methylation (Freitag et al. 2004; Honda and Selker 2008). To confirm the association between DDB1 and ERI-1, we performed coimmunoprecipitation in the wild-type, eri$1^{K O}$;Flag-ERI-1, and ERI-1 ${ }^{K O}{ }_{\text {; Flag-ERI-1 }}{ }^{\triangle \mathrm{RRM}}$ strains with the Flag monoclonal antibody. As shown in Figure 6A, Flag-ERI-1 precipitated DDB1 in both eri-1 ${ }^{\mathrm{KO}}$;Flag-ERI-1 and $e r i-1^{K O}$; Flag-ERI- $1^{\triangle \mathrm{RRM}}$ strains but not in the wildtype strain, indicating that ERI-1 interacts with DDB1 independently of its RNA-binding domain. This suggests that ERI-1 recruits the DCDC complex to mediate $\mathrm{H} 3 \mathrm{~K} 9 \mathrm{me} 3$ and then DNA methylation. In support of this, we found that DLDM was completely abolished in the $d d b 1^{K O}$ mutant (Fig. 6B). DLDM was also abolished in the $\operatorname{dim}-5^{K O}$ (which does not express the H3K9 methyltransferase) and dim- $2^{K O}$ (which does not express the DNA methyltransferase) strains.

We then analyzed whether the ERI-1-mediated histone and DNA methylation silences transcription at the disiRNA loci. We performed RT-qPCR to measure mRNA levels produced from four disiRNA loci. As shown in Figure 6C, mRNA levels from the disiRNA-6, disiRNA-8, and disiRNA-29 loci were significantly elevated in the DNA methylation-deficient mutants $\left(\operatorname{dim}-5^{\mathrm{KO}}\right.$, dim$2^{K O}$, and $\left.d d b 1^{K O}\right)$, suggesting that DLDM does indeed inhibit transcription. However, the levels of frq (disiRNA47) were not significantly affected in any of these strains, likely due to the compensatory effect of a circadian negative feedback loop in which FRQ represses its own transcription (Dunlap 2006; Heintzen and Liu 2007).

\section{ERI-1 mediates post-transcriptional RNA degradation in the cytoplasm}

In a wild-type strain, most of the ERI-1-targeted mRNAs do not interact with ERI-1 in the nucleus and are not involved in DLDM, suggesting that ERI-1 also has a post-transcriptional function in the cytoplasm. Consistent with this notion, we found that ERI-1 is mostly a cytoplasmic protein (Supplemental Fig. S2). Since ERI-1 is an exonuclease, we examined whether ERI-1 regulates its target mRNA levels. Using HITS-CLIP, we selected 18 ERI-1 target mRNAs and four mRNAs without ERI-1binding sites and compared their mRNA levels in the wild-type and eri-1 ${ }^{K O}$ strains. As shown in Figure 7A, 16 of the 18 ERI-1-targeted mRNA levels were significantly higher in the eri-1 ${ }^{K O}$ mutant than in the wild-type strain. In contrast, the levels of mRNAs not targeted by ERI-1 were unchanged in the eri- $1^{K O}$ mutant.

To further understand the role of ERI-1, we used strandspecific RT-qPCR and Northern blot analyses to determine the levels of fr $q$ in the eri-1 $1^{K O}$ and wild-type strains. As expected, the fr $q$ mRNA level was significantly elevated in the eri- $1^{K O}$ mutant compared with that in the wildtype strain (Fig. 7B). The increase in frq mRNA should be 


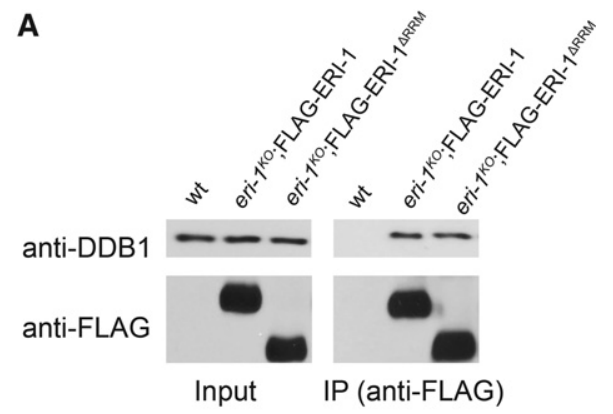

B

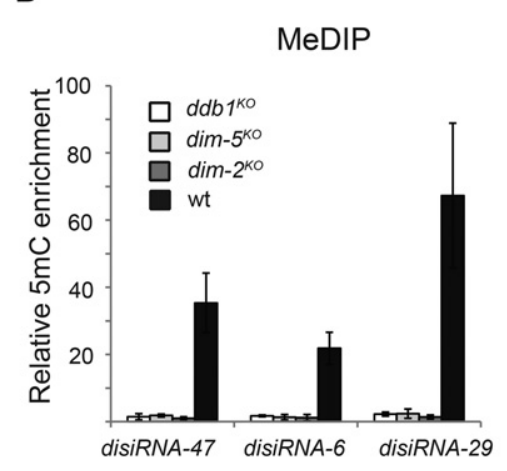

\section{C}

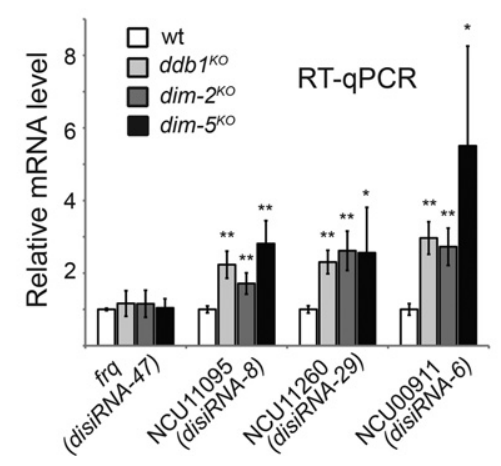

Figure 6. DDB1 is an ERI-1-associated protein and is required for DLDM. (A) Immunoprecipitation with Flag antibodies showing the association between ERI-1 (Flag-ERI-1 or Flag-ERI-1 ${ }^{\triangle R R M}$ ) and the endogenous DDB1. The antibodies used in the Western analyses are indicated at the left. (B) MeDIP results show the lack of DLDM levels in the $d d b 1^{K O}, \operatorname{dim}-5^{K O}$, and $\operatorname{dim}-2^{K O}$ strains. (C) RT-qPCR results showing the mRNA levels of selected disiRNA loci in the $d d b 1^{K O}, \operatorname{dim}-5^{K O}$, and dim- $2^{K O}$ strains. (*) $\left.P<0.05 ;{ }^{* *}\right) P<0.01$, Student's $t$-test.

due to the cytoplasmic role of ERI-1. This effect is largely independent of DLDM because fr $q$ mRNA levels were not altered relative to control levels when DLDM was abolished (Fig. 6C). This result is also consistent with an increase of FRQ protein level in the eri-1 ${ }^{K O}$ mutant (Supplemental Fig. S6), suggesting that ERI-1 has a function in the Neurospora circadian clock. Indeed, race tube assays monitoring the circadian conidiation rhythms showed that the eri-1 ${ }^{K O}$ mutant exhibited a shorter-peri- od circadian rhythm than the wild-type strain $(21.02 \mathrm{~h} \pm$ $0.21 \mathrm{~h}$ vs. $22.08 \mathrm{~h} \pm 0.13 \mathrm{~h}$ ) (Fig. 7C). As expected, this period phenotype was rescued by the expression of the wildtype ERI-1 but not by expression of the RRM deletion or catalytically dead ERI-1.

The high levels of mRNA in the eri-1 ${ }^{K O}$ mutant suggest that ERI-1 is a nuclease that mediates mRNA degradation. Supporting this hypothesis, we found that the rate of $f r q$ mRNA degradation after the addition of the transcription inhibitor thiolutin was significantly slower in the eri-1 ${ }^{K O}$ mutant than that in the wild-type strain (Fig. 7D). Together, these results indicate that ERI-1 is an important regulator of RNA metabolism in the cytoplasm. It likely regulates RNA stability through its exonuclease activity.

\section{Discussion}

In this study, we showed that ERI-1 is a critical component of DLDM and disiRNA production. By studying ERI-1, we established a mechanism by which antisense transcription results in epigenetic gene silencing by regulating transcription kinetics and also revealed how ERI-1 functions as a post-transcriptional regulator of RNA metabolism.

Our results suggest that antisense transcription results in RNA Pol II stalling, which allows ERI-1 to specifically recognize nascent RNAs with ERI-1-binding sites and mediate histone and DNA methylation of nearby chromatin (Fig. 7E). In this model, the ERI-1-binding sites on mRNA and the presence of convergent transcription are required for DLDM. This model is supported by several lines of evidence. First, DLDM is dramatically impaired in the eri$1^{K O}$ mutant. Although HITS-CLIP experiments showed that ERI-1 recognizes G-rich motifs present in $3^{\prime}$ UTRs of hundreds of mRNAs, DLDM was observed only at the loci where antisense transcription occurs. Importantly, in the nucleus, ERI-1 interacts only with RNAs from the disiRNA loci but not those from non-disiRNA loci (Fig. 4).

Second, we showed previously that antisense transcription induces DLDM from an artificial locus and the disiRNA-47 ( $f r q$ ) locus (Belden et al. 2011; Dang et al. 2013; Li et al. 2015). Here we showed that the mutation of ERI-1-binding sites on qrf RNA severely impaired DLDM at the frq locus (Fig. 3G). In addition, for the loci that lack DLDM but produce mRNAs with ERI-1-binding sites, introduction of antisense transcription was sufficient to trigger DNA methylation (Fig. 4D). Moreover, antisense transcription specifically induced ERI-1 association with RNA containing ERI-1-binding sites in the nucleus (Fig. 4G). Together, these results demonstrate that ERI-1-binding sites on mRNA sequences and antisense transcription are both necessary and sufficient for histone and DNA methylation at the nearby DNA.

Third, our results demonstrate that antisense transcription acts as a mechanism to distinguish nascent RNAs encoded by disiRNA loci from those encoded by nondisiRNA loci. Antisense transcription has been shown to result in RNA Pol II stalling during elongation (Hobson et al. 2012; Xue et al. 2014). When a gene encoding a 
A

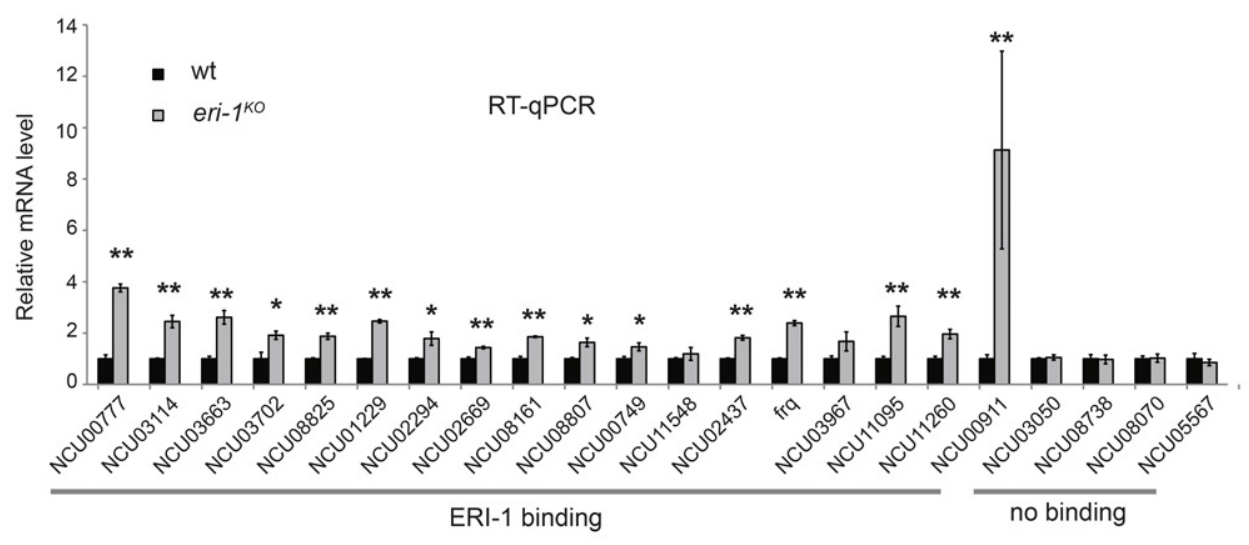

B

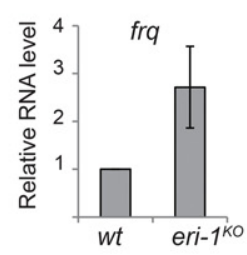

frq

rRNA

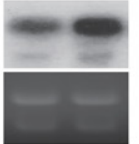

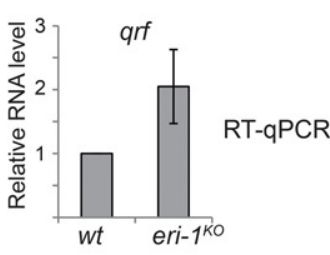

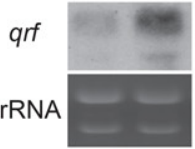

northern

C

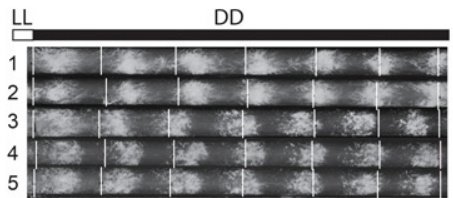

\begin{tabular}{|c|c|c|}
\hline No. & Strain & Period (hr) \\
\hline 1 & wt & $22.08 \pm 0.13$ \\
\hline 2 & eri-1 ${ }^{\mathrm{KO}}: \mathrm{MYC}-\mathrm{ERI}-1$ & $22.21 \pm 0.28$ \\
\hline 3 & eri-1 $1^{\text {ко }}$ & $21.02 \pm 0.21$ \\
\hline 4 & eri-1 ${ }^{K O}: M Y C-E R I-1 \triangle R R M$ & $20.94 \pm 0.06$ \\
\hline 5 & eri-1 ${ }^{\text {KO:}: M Y C-E R I-1 C D ~}$ & $20.88 \pm 0.37$ \\
\hline
\end{tabular}

E

D Time after thiolutin treatment $(\mathrm{min})$
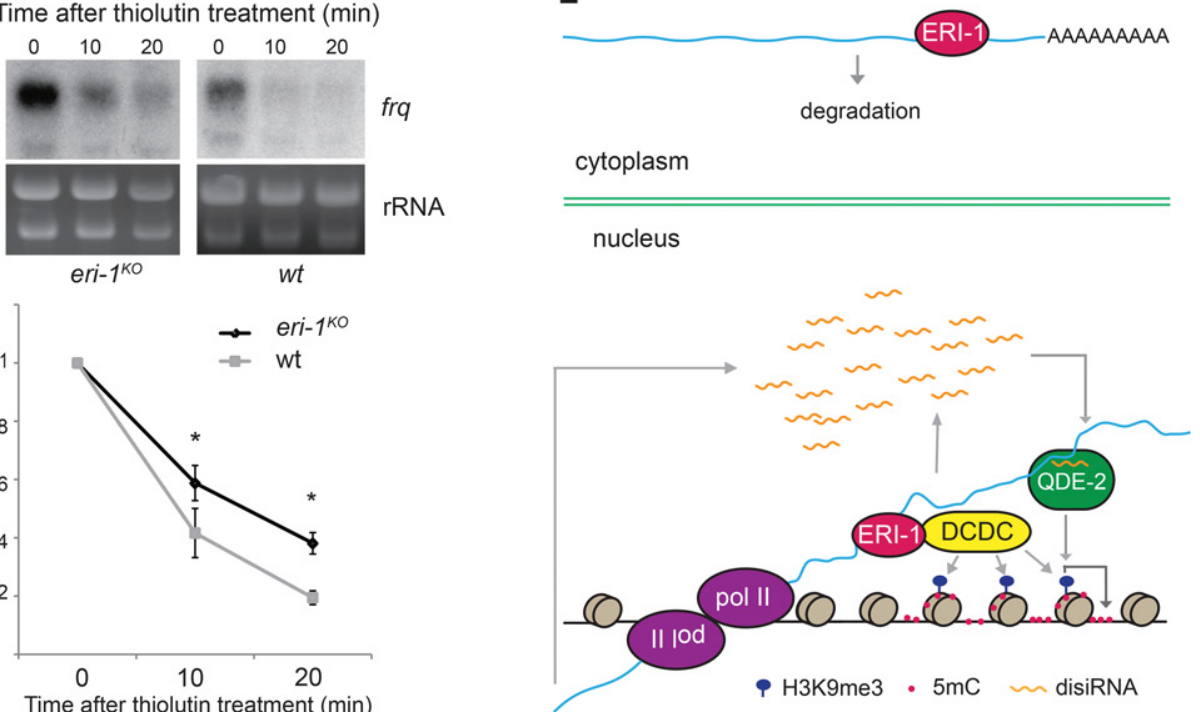

Figure 7. ERI-1 regulates target mRNA levels in the cytoplasm. $(A)$ RT-qPCR results showing the levels of selected ERI-1-targeted RNAs in the eri-1 ${ }^{K O}$ and wild-type strains. The mRNA levels from the wild type are set as 1 . Three independent repeats were performed, and the values represent mean $\left.\pm \mathrm{SD} .\left({ }^{*}\right) P<0.05 ;{ }^{* *}\right) P<0.01$, Student's $t$-test). $(B)$ RNA levels of frq and $q r f$ as determined by strand-specific RTqPCR (top panels) and Northern blot analysis (middle panels). Agarose gels stained with ethidium bromide indicate the RNA loading of Northern blots. $(C)$ Race tube analyses showing the circadian conidiation rhythms of the indicated strains. Three independent repeats were performed, and period determination results are shown in the bottom panel. $(D)$ Northern blot analysis showing that frq mRNA is more stable after thiolutin treatment in the eri-1 ${ }^{K O}$ strain than in the wild-type strain. The cultures were treated with thiolutin and collected at the indicated time points. Three independent repeats were performed, and one representative result is shown in the top panel. The values in the bottom panel represent mean $\pm \mathrm{SD}$. $\left(^{*}\right) P<0.05$, Student's $t$-test. $(E)$ A model illustrating the function of ERI-1 in regulating target RNA stability in the cytoplasm and mediating histone and DNA methylation in the nucleus in the presence of antisense transcription. 
subunit of the Pafl complex, which regulates Pol II elongation and termination, was deleted, we observed Pol II stalling and DNA methylation at some loci that produce mRNAs with ERI-1-binding sites but lack antisense transcription (Fig. 5). These results strongly suggest that the role of antisense transcription is to slow down transcription elongation or termination, thus providing more time for nascent RNAs with the ERI-1-binding sites to be recognized by ERI-1. In the absence of antisense transcription, mRNAs are quickly processed and transported into the cytoplasm before they can be recognized by ERI-1 in the nucleus. Therefore, the decelerated transcription kinetics that results from antisense transcription is the mechanism that allows ERI-1 to distinguish RNAs from disiRNA loci from non-disiRNA loci. In fision yeast, the Paf1 complex promotes efficient transcription termination and is a negative regulator of RNAi-directed heterochromatin formation (Kowalik et al. 2015). Therefore, in both Neurospora and fission yeast, transcription kinetics plays an important role in determining the recognition of nascent transcripts by chromatin-modifying factors.

Finally, we showed that ERI-1 physically associates with DDB1, a core component of that DCDC complex that is responsible for $\mathrm{H} 3 \mathrm{~K} 9 \mathrm{me} 3$ (Lewis et al. 2010; Zhao et al. 2010). In Neurospora, H3K9me3 can recruit HP1 and the DNA methyltransferase DIM-2, which eventually lead to DNA methylation (Freitag et al. 2004; Honda and Selker 2008; Rountree and Selker 2010).

In addition to its role in transcriptional gene silencing, we showed that ERI-1 is also an important regulator of RNA stability. The vast majority of the ERI-1targeted mRNAs is produced from loci without antisense transcription and is not a target of ERI-1 in the nucleus. Levels of most of the examined ERI-1 target mRNAs are significantly elevated in the eri-1 ${ }^{K O}$ strains (Fig. 7). We also found that the levels of $f_{r} q$ mRNA and FRQ proteins were significantly elevated in the eri- $1^{K O}$ strain. As the eri-1 ${ }^{K O}$ strain exhibited a short-period circadian clock rhythm, our data are consistent with a role for ERI-1 in the circadian clock. Furthermore, fr $q$ mRNA degradation was impaired in the eri-1 ${ }^{K O}$ mutant, suggesting that ERI-1 acts a ribonuclease that can degrade its mRNA targets. Consistent with this notion, ERI-1 mutations of its putative catalytic sites abolished its function (Figs. 3B, 7C). However, we were not able to detect significant ribonuclease activity using recombinant ERI-1 expressed and purified from Escherichia coli. This failure may have been due to misfolding of the recombinant protein, or the nuclease activity of ERI-1 might require another protein, suggesting a conserved function of ERI-1 in mammals. Its human homolog also binds to the $3^{\prime}$ end of histone-encoding mRNA and may regulate its stability (Dominski et al. 2003; Yang et al. 2006).

ERI-1 was critical to both DLDM and disiRNA production. Although disiRNA contributes to DLDM and mediates QDE-2-dependent DNA methylation, it is not essential for DLDM, since DLDM is maintained in the qde-2-null mutant and $q$ de- $2^{R I P} ; s m s-2^{K O}$ mutant (Fig. 2; Supplemental Figs. S1, S3). How QDE-2 and disiRNA mediate DNA methylation is presently not clear. It is likely that they act in a mechanism that is similar to siRNAmediated heterochromatin formation in $S$. pombe by recognizing the nascent RNA and recruiting histone-modifying enzymes (Volpe et al. 2002; Buhler and Moazed 2007; Grewal 2010; Halic and Moazed 2010). Consistent with this notion, we found that transcription at a targeted locus is required for disiRNA-mediated DNA methylation (Fig. 2D). Although how ERI-1 functions in the disiRNA production pathway is not known, it may act via its ribonuclease function in the nucleus. Even though ERI-1 was initially identified in C. elegans as an exonuclease that suppresses exogenous dsRNA-mediated RNAi, it was later shown to be required for the production of endo-siRNAs (Kennedy et al. 2004; Duchaine et al. 2006; Iida et al. 2006). Thus, ERI-1 family proteins appear to have a conserved role in sRNA biogenesis in eukaryotes.

\section{Materials and methods \\ Strains and culture conditions}

In this study, N. crassa strain FGSC $4200(a)$ was used as the wildtype strain. Mutant strains $q$ de $-2^{R I P}, \operatorname{dim}-2^{K O}$, and dim- $5^{K O}$ were generated in previous studies (Choudhary et al. 2007; Zhao et al. 2010). The knockout strains were obtained from the Neurospora Knockout Consortium (Colot et al. 2006). The eri-1 ${ }^{K O} ; v v d^{K O}$ strain was generated by crossing eri-1 ${ }^{K O}$ (FGSC12630, a) with the $v v d^{K O}$ (FGSC11556, A) strain. The eri-1 ${ }^{\text {KO }}$ (his-3), vvd ${ }^{\text {KO }}$ (his-3), and $c d c 73^{K O}$ (his-3) strains were generated by crossing the 301-6 strain (bd, his-3, A) with the eri1 ${ }^{K O}$ (FGSC12630, a), $v v d^{K O}(\mathrm{FGSC} 11555, a)$, and $c d c 73^{K O}(\mathrm{FGSC} 20287, a)$ strains, respectively. The eri-1 ${ }^{K O}$ (his-3), vvd $d^{K O}$ (his-3), 301-6, 303-3 (bd, frq ${ }^{10}$, and his-3) (Cha et al. 2011), and qde-2 ${ }^{R I P}$ (his-3) strains (Choudhary et al. 2007) were the host strains for his-3 targeting constructs. The constructs expressing Flag-tagged and Myc-tagged protein were driven by $c c g-1$ and $q a-2$ promoters, respectively.

Culture conditions have been described previously (Aronson et al. 1994). Neurospora mats were cut into discs and transferred to flasks with minimal medium ( $1 \times$ Vogel's, $2 \%$ glucose). After 24 $\mathrm{h}$, the tissues were harvested. To induce the $q a-2$ promoter, liquid cultures were grown in 10 mM QA (pH 5.8), $1 \times$ Vogel's, $0.1 \%$ glucose, and $0.17 \%$ arginine. The tissues were harvested after 24 h. Race tube medium contained $1 \times$ Vogel's, $0.1 \%$ glucose, $0.17 \%$ arginine, $50 \mathrm{ng} / \mathrm{mL}$ biotin, and $1.5 \%$ agar.

\section{DNA methylation analyses}

DNA preparation, DNA methylation-specific qPCR, Southern blot analyses, and MeDIP were performed as described in a previous study (Dang et al. 2013). In Southern blot and MeDIP analyses, $10 \mu \mathrm{g}$ of DNA was used. A mouse anti-5mC monoclonal antibody (Active Motif, catalog no. 39649) was used for MeDIP, and the am locus was used as negative control.

MeDIP-seq analyses were performed as described previously (Pomraning et al. 2009) by using the NEBNext DNA library preparation kit (New England Biolabs, catalog no. E6040). Briefly, DNA samples were sonicated, and the fraction of $\sim 200$ base pairs (bp) was recovered from an agarose gel. Approximately $500 \mathrm{ng}$ of sheared DNA was used for end repair, dA-tailing, adaptor ligation, and $U$ excision as described in the kit manual. MeDIP was performed with the ligation products and enriched by PCR as 
described in the kit manual. Illumina sequencing was performed using an Illumina HiSeq 2000 platform.

The raw MeDIP-seq data were aligned against the $N$. crassa genome (version 10; N. crassa Database, Broad Institute) with Bowtie (version 1.1.1). Two mismatches were allowed, and the setting -m 1 -best -strata was used to allow only one best hit for each read. PCR duplicates were then removed from alignment results with Samtools (Li et al. 2009). The bedgraph files were generated with BEDTools for visualization on IGV (Quinlan and Hall 2010; Robinson et al. 2011), and mapped reads were normalized to equal library size (reads per million [RPM]) to facilitate comparison between samples. The dim-2 ${ }^{K O}$ strain was set as the control.

\section{RNA analyses}

RNA extraction, RT-qPCR, strand-specific RT-qPCR, and Northern blot were performed as described previously (Xue et al. 2014). $\beta$-Tubulin was used as internal control. The mRNA decay assay was performed as described previously (Guo et al. 2009). The tissues were harvested at different time points after the addition of thiolutin (final concentration $12 \mu \mathrm{g} / \mathrm{mL}$ ), and Northern analyses were performed.

\section{SRNA analyses}

sRNA Northern analyses were performed as described previously (Lee et al. 2010). RNA from a 25- $\mu$ g sample was separated on a $16 \%$ denaturing polyacrylamide gel containing urea and transferred onto a Hybond-NX membrane (GE Healthcare). 1-Ethyl3-(3-dimethylaminopropyl) carbodiimide (EDC) was used to cross-link the membrane (Pall and Hamilton 2008).

Total RNA of the eri1 ${ }^{K O}$ strain and wild-type strain were extracted with TRIzol reagent. sRNA enrichment and sRNA sequencing library construction and data analyses were performed as described previously (Lee et al. 2010; Dang et al. 2013).

\section{Immunoprecipitation and mass spectrometry}

Tissue samples grown for $\sim 2 \mathrm{~d}$ at room temperature were harvested, pulverized, and suspended overnight in purification buffer (150 mM HEPES at $\mathrm{pH} 7.4,250 \mathrm{mM} \mathrm{KCl}, 1 \mathrm{mM}$ EDTA, $1 \mathrm{mM}$ DTT, $10 \%$ glycerol, protease inhibitor) with anti-Flag M2 magnetic beads (Sigma). The beads were washed six times with purification buffer, and bound proteins were eluted with $200 \mu \mathrm{g} / \mathrm{mL}$ Flag tag peptide (Sigma). Eluted proteins were concentrated with TCA precipitation, resolved on SDS-PAGE, and recovered for mass spectrometry analysis. To verify the mass spectrometry results, Neurospora mats were cut into discs and transferred to flasks with minimal medium ( $1 \times$ Vogel's, $\%$ glucose). After growing for $24 \mathrm{~h}$ at room temperature, the tissues were briefly cross-linked with formaldehyde ( $1 \%$ final concentration) for 15 min, stopped with $125 \mathrm{mM}$ glycine, washed with $1 \times$ PBS, and harvested. Protein extraction and quantification, Western blot analysis, and immunoprecipitation assays were performed as described previously (Cheng et al. 2001). Lysates ( $2 \mathrm{mg}$ ) were incubated overnight with anti-Flag M2 magnetic beads (Sigma) and washed six times with wash buffer (same as lysis buffer except with $500 \mathrm{mM} \mathrm{NaCl}$ ), and the elute proteins were analyzed with Western blot.

ChIP assay and ChIP-seq (ChIP combined with high-throughput sequencing) analyses

ChIP assays were performed as described previously (Dang et al. 2013). In each reaction, $2 \mathrm{mg}$ of total lysate and $2 \mu \mathrm{L}$ of Pol II
CTD (Abcam) or H3K9me3 (Abcam, ab8898) were used. Each experiment was performed independently three times, and immunoprecipitation with IgG was used as the negative control. Immunoprecipitated DNA was quantified using real-time PCR.

\section{Chromatin-associated RNA assay}

The method was adapted as described previously (Bhatt et al. 2012). Specifically, $\sim 7 \mathrm{~mL}$ of tissue powder was dissolved in 15 $\mathrm{mL}$ of buffer A [1 M sorbitol, $7 \%$ ficoll, $20 \%$ glycerol, $5 \mathrm{mM} \mathrm{Mg}$ $(\mathrm{OAc})_{2}, 3 \mathrm{mM} \mathrm{CaCl}_{2}, 50 \mathrm{mM}$ Tris- $\mathrm{HCl}$ at $\mathrm{pH}$ 7.5] and gently mixed for $5 \mathrm{~min}$ at $4^{\circ} \mathrm{C}$. Large cell debris was removed with centrifugation at $3000 \mathrm{~g}$ for $10 \mathrm{~min}$ at $4^{\circ} \mathrm{C}$. Supernatant was filtered by four layers of cheese cloth and further centrifuged at $12,000 \mathrm{~g}$ for 5 $\mathrm{min}$. The pellets were washed once with $2 \mathrm{~mL}$ of buffer A and gently resuspended in $1000 \mu \mathrm{L}$ of cold glycerol buffer $(20 \mathrm{mM}$ Tris at $\mathrm{pH} 7.9,75 \mathrm{mM} \mathrm{NaCl}, 0.5 \mathrm{mM}$ EDTA, $50 \%$ glycerol, 0.85 mM DTT). One-hundred microliters of supernatant was saved for DNA extraction, which served as the loading control. The remaining $900 \mu \mathrm{L}$ of supernatant was mixed with an equal volume of cold nucleus lysis buffer (20 mM HEPES at $\mathrm{pH} 7.6,7.5 \mathrm{mM}$ $\mathrm{MgCl}_{2}, 0.2 \mathrm{mM}$ EDTA, $0.3 \mathrm{M} \mathrm{NaCl}, 1 \mathrm{M}$ urea, $1 \%$ NP-40, 1 $\mathrm{mM}$ DTT), incubated for $1 \mathrm{~min}$ on ice, and centrifuged at $20,000 \mathrm{~g}$ for $2 \mathrm{~min}$ at $4^{\circ} \mathrm{C}$. The pellet (the chromatin fraction) was resuspended with $100 \mu \mathrm{L}$ of cold $1 \times$ PBS, and nascent RNA associated with chromatin were purified with TRIzol reagent. For each sample, equal volumes of RNA extracts $(\sim 500 \mathrm{ng}$ to $1 \mu \mathrm{g}$ ) were used for RT-qPCR. The RT-qPCR results were normalized with qPCR results from the loading control (i.e., total DNA/ chromatin input).

\section{RNA immunoprecipitation assay}

The RNA immunoprecipitation assay was performed as described previously (Niranjanakumari et al. 2002). Briefly, Neurospora was cultured in flasks for $48 \mathrm{~h}$, and then formaldehyde was added to the medium to a final concentration of $1 \%(\mathrm{v} / \mathrm{v})$. After incubation at room temperature for $15 \mathrm{~min}$ with slow shaking, the reaction was quenched with $0.1 \mathrm{M}$ glycine (final concentration) for $5 \mathrm{~min}$ and then harvested under vacuum. After washing with ice-cold washing buffer (50 mM HEPES at pH 7.4, $137 \mathrm{mM}$ $\mathrm{NaCl}$ ), the tissues were ground in liquid nitrogen.

For RNA immunoprecipitation of total cell extracts, $\sim 400 \mu \mathrm{L}$ of tissue powder was suspended in $1 \mathrm{~mL}$ of ice-cold RIP lysis buffer (50 mM HEPES at $\mathrm{pH} 7.4,150 \mathrm{mM} \mathrm{NaCl}, 1.5 \mathrm{mM} \mathrm{MgCl}_{2}, 1 \% \mathrm{NP}-$ 40, $0.05 \%$ SDS, 1 mM EDTA) supplemented with protease inhibitor and RNase Out. The samples were gently sonicated to increase solubility. For nuclear RNA immunoprecipitation, nuclei were first purified as described previously (Xue et al. 2014), resuspended RIP lysis buffer, and sonicated. The supernatant was collected, $5 \mathrm{mg}$ of protein (from total cell extracts) or $2 \mathrm{mg}$ of protein (from nuclear extracts) was added to $1 \mathrm{~mL}$ of ice-cold RIP lysis buffer, and $35 \mu \mathrm{L}$ of Flag resin suspension (anti-Flag M2 magnetic beads, Sigma) was added to the buffer. Samples were rotated for $12 \mathrm{~h}$ at $4^{\circ} \mathrm{C}$. The beads were washed thoroughly with RIP washing buffer (50 mM HEPES at pH 7.4, $500 \mathrm{mM} \mathrm{NaCl}, 1.5 \mathrm{mM}$ $\mathrm{MgCl}_{2}, 1 \%$ NP-40, $0.1 \%$ SDS, 1 mM EDTA). The beads were resuspended in $300 \mu \mathrm{L}$ of RIP lysis buffer supplemented with 100 $\mu \mathrm{g} / \mathrm{mL} 3 \times$ Flag-peptide (Sigma) and then rotated for $30 \mathrm{~min}$ at room temperature. The buffer was collected, and $3 \mu \mathrm{L}$ of $5 \mathrm{M}$ $\mathrm{NaCl}$ and $1 \mu \mathrm{g}$ of proteinase $\mathrm{K}$ were added. After $1 \mathrm{~h}$ at $42^{\circ} \mathrm{C}$, samples were incubated for $1 \mathrm{~h}$ at $65^{\circ} \mathrm{C}$ to reverse the cross-linking. The RNA was precipitated and treated with DNase I. The levels of mRNA were determined by RT-qPCR with no-tag control (wild-type strain) set as 1 . 


\section{HITS-CLIP}

The CLIP procedure was performed essentially as described previously (Becht et al. 2006; Darnell 2012). Briefly, Neurospora was cultivated in large plates for $24 \mathrm{~h}$ at room temperature, and the plates were irradiated on ice with $480 \mathrm{~mJ} / \mathrm{cm}^{2}$ in a UV box. The Neurospora tissue was harvested and ground in liquid nitrogen. About $150 \mu \mathrm{L}$ of tissue powder was suspended in $1.2 \mathrm{~mL}$ of ice-cold RIP lysis buffer (1 mM DTT, protease inhibitor, RNase Out) and vortexed at high speed. The tubes were rotated for 20 min at $4^{\circ} \mathrm{C}$. RQ1 DNase (1:50; Promega) was added, and samples were incubated for $10 \mathrm{~min}$ at $37^{\circ} \mathrm{C}$. After gentle sonication, samples were centrifuged at $12,000 \mathrm{~g}$. Next, $35 \mu \mathrm{L}$ of Flag resin suspension (anti-Flag M2 magnetic beads, Sigma) in RIP buffer was added to the supernatants, and samples were incubated for $12 \mathrm{~h}$ at $4^{\circ} \mathrm{C}$. Beads were washed six times with RIP washing buffer and twice with PNK buffer (50 mM Tris- $\mathrm{HCl}$ at $\mathrm{pH} 7.4,5 \mathrm{mM}$ $\mathrm{MgCl}_{2}, 0.5 \% \mathrm{NP}-40$ ). Beads were resuspended in $300 \mu \mathrm{L}$ of PNK buffer with $50 \mathrm{U}$ of RNase $\mathrm{T} 1$ and incubated for $10 \mathrm{~min}$ at $37^{\circ} \mathrm{C}$. Beads were washed five times with PNK buffer, mixed with CIP (New England Biolabs) reaction system ( $3 \mu \mathrm{L}$ of CIP, $40 \mathrm{U}$ of RNase In [Promega] in $80 \mu \mathrm{L}$ of dephosphorylation buffer), and then incubated for $30 \mathrm{~min}$ at $37^{\circ} \mathrm{C}$. Beads were washed twice with PNK buffer supplemented with $20 \mathrm{mM}$ EGTA, twice with PNK buffer, and twice with $0.1 \mathrm{mg} / \mathrm{mL}$ BSA in RNase-free water and then resuspended in $50 \mu \mathrm{L}$ of T4 RNA ligation buffer with 5 $\mu \mathrm{M} \mathrm{3} 3^{\prime}$ adaptor (New England Biolabs, E7300S), 10\% PEG 8000, and $40 \mathrm{U}$ of RNase Out. Finally, $300 \mathrm{U}$ of T4 RNA ligase 2 was added, and samples were incubated for $3 \mathrm{~h}$ at room temperature. Beads were washed five times with PNK buffer and added to $100 \mu \mathrm{L}$ of T4 PNK reaction buffer A with $20 \mathrm{U}$ of T4 PNK, 1 mM ATP, and $40 \mathrm{U}$ of RNase Out, and then the beads were incubated for $30 \mathrm{~min}$ at $37^{\circ} \mathrm{C}$ with rotation. Beads were washed twice with PNK buffer supplemented with 20 mM EGTA, twice with PNK buffer, and twice with Proteinase $\mathrm{K}$ buffer $(50 \mathrm{mM}$ Tris- $\mathrm{HCl}$ at $\mathrm{pH} 7.4,75 \mathrm{mM} \mathrm{NaCl}, 1 \% \mathrm{SDS}, 1 \mathrm{mM}$ EDTA); resuspended in $400 \mu \mathrm{L}$ of Proteinase K buffer; and boiled for $5 \mathrm{~min}$ at $95^{\circ} \mathrm{C}$. The eluted RNA-protein complexes were collected and treated with $1.0 \mathrm{mg} / \mathrm{mL}$ Proteinase $\mathrm{K}$ with RNase Out for $2 \mathrm{~h}$ at $55^{\circ} \mathrm{C}$. The RNA was precipitated with $1 \mu \mathrm{L}$ of RT primer (New England Biolabs, E7300S), dissolved in $25.5 \mu \mathrm{L}$ of nuclease-free water, heated for $5 \mathrm{~min}$ at $75^{\circ} \mathrm{C}$, and then transferred for $15 \mathrm{~min}$ to $37^{\circ} \mathrm{C}$ followed by $15 \mathrm{~min}$ at $25^{\circ} \mathrm{C}$. Denatured $5^{\prime} \mathrm{SR}$ adaptor for Illumina was added according to the manufacturer's manual (New England Biolabs, E7300S), and first strand cDNA was synthesized using ProtoScript II reverse transcriptase. PCR was performed followed by the addition of SR Primer for Illumina, index primers (six and 12 were used in this study), and LongAmp Taq $2 \times$ master mix. The PCR product was precipitated, dissolved in $25 \mu \mathrm{L}$ of nuclease-free water, and then separated on $8 \%$ PAGE. The target bands (140-150 bp) were recovered from the gel for sequencing.

The raw CLIP-seq data (NCBI accession no. PRJNA350329) were aligned against the $N$. crassa genome (version 10, N. crassa Database, Broad institute) with TopHat (version 2.0.11). A new annotation of transcriptome was created based on the version 10 release of annotation and RNA-seq results to correct the ranges of some transcripts. The bedgraph files were generated with BEDTools for visualization on IGV. The aligned results were uploaded to the PIPE-CLIP Galaxy site (http://pipeclip.qbrc.org) to perform peak calling and cross-link site analyses with default settings (Chen et al. 2014). For analyses with dCLIP software (version 1.6.4), "all" was chosen for the -mut option; otherwise, default settings were used (Wang et al. 2014). To further validate the peak calling results, we selected only peaks with a quality score $\geq 5$, peak length $\geq 50 \mathrm{bp}$, summit of peaks $\geq 10$ (RPM), and peak densities at least fivefold higher than that of the control sample (wild-type strain, no Flag-ERI-1). The results are in Supplemental Table S1, and the scripts used for the analyses are available on request.

\section{Acknowledgments}

We thank Zhihong Xue and Wei Guo during the early stage of this project, and other laboratory members for technical assistance and discussion. This work was supported by grants from the National Institutes of Health and the Welch Foundation (I-1560) to Y.L., and a scholarship from the Chinese Scholarship Council (no. 2011842283) awarded to J.C.

\section{References}

Aronson B, Johnson K, Loros JJ, Dunlap JC. 1994. Negative feedback defining a circadian clock: autoregulation in the clock gene frequency. Science 263: 1578-1584.

Arthanari Y, Heintzen C, Griffiths-Jones S, Crosthwaite SK. 2014. Natural antisense transcripts and long non-coding RNA in Neurospora crassa. PLoS One 9: e91353.

Bailey TL, Boden M, Buske FA, Frith M, Grant CE, Clementi L, Ren JY, Li WW, Noble WS. 2009. MEME SUITE: tools for motif discovery and searching. Nucleic Acids Res 37: W202-W208.

Becht P, Konig J, Feldbrugge M. 2006. The RNA-binding protein Rrm4 is essential for polarity in Ustilago maydis and shuttles along microtubules. J Cell Sci 119: 4964-4973.

Belden WJ, Lewis ZA, Selker EU, Loros JJ, Dunlap JC. 2011. CHD1 remodels chromatin and influences transient DNA methylation at the clock gene frequency. PLoS Genet 7: e1002166.

Bhatt DM, Pandya-Jones A, Tong AJ, Barozzi I, Lissner MM, Natoli G, Black DL, Smale ST. 2012. Transcript dynamics of proinflammatory genes revealed by sequence analysis of subcellular RNA fractions. Cell 150: 279-290.

Buhler M, Moazed D. 2007. Transcription and RNAi in heterochromatic gene silencing. Nat Struct Mol Biol 14: 1041-1048.

Camblong J, Iglesias N, Fickentscher C, Dieppois G, Stutz F. 2007. Antisense RNA stabilization induces transcriptional gene silencing via histone deacetylation in $S$. cerevisiae. Cell 131: 706-717.

Cha J, Yuan H, Liu Y. 2011. Regulation of the activity and cellular localization of the circadian clock protein FRQ. J Biol Chem 286: 11469-11478.

Chang SS, Zhang Z, Liu Y. 2012. RNA interference pathways in fungi: mechanisms and functions. Annu Rev Microbiol 66: 305-323.

Chen BB, Yun J, Kim MS, Mendell JT, Xie Y. 2014. PIPE-CLIP: a comprehensive online tool for CLIP-seq data analysis. Genome Biol 15: R18.

Cheng P, Yang Y, Heintzen C, Liu Y. 2001. Coiled-coil domainmediated FRQ-FRQ interaction is essential for its circadian clock function in Neurospora. EMBO J 20: 101-108.

Choudhary S, Lee HC, Maiti M, He Q, Cheng P, Liu Q, Liu Y. 2007. A double-stranded-RNA response program important for RNA interference efficiency. Mol Cell Biol 27: 3995-4005.

Colot HV, Park G, Turner GE, Ringelberg C, Crew CM, Litvinkova L, Weiss RL, Borkovich KA, Dunlap JC. 2006. A highthroughput gene knockout procedure for Neurospora reveals functions for multiple transcription factors. Proc Natl Acad Sci 103: 10352-10357. 
Dang Y, Li L, Guo W, Xue Z, Liu Y. 2013. Convergent transcription induces dynamic DNA methylation at disiRNA loci. PLoS Genet 9: e1003761.

Darnell R. 2012. CLIP (cross-linking and immunoprecipitation) identification of RNAs bound by a specific protein. Cold Spring Harb Protoc 2012: 1146-1160.

David L. 2006. A high-resolution map of transcription in the yeast genome. Proc Natl Acad Sci 103: 5320-5325.

Deuve JL, Avner P. 2011. The coupling of X-chromosome inactivation to pluripotency. Annu Rev Cell Dev Biol 27: 611-629.

Dominski Z, Yang XC, Kaygun H, Dadlez M, Marzluff WF. 2003. A $3^{\prime}$ exonuclease that specifically interacts with the $3^{\prime}$ end of histone mRNA. Mol Cell 12: 295-305.

Donaldson ME, Saville BJ. 2012. Natural antisense transcripts in fungi. Mol Microbiol 85: 405-417.

Duchaine TF, Wohlschlegel JA, Kennedy S, Bei Y, Conte D Jr, Pang K, Brownell DR, Harding S, Mitani S, Ruvkun G, et al. 2006. Functional proteomics reveals the biochemical niche of C. elegans DCR-1 in multiple small-RNA-mediated pathways. Cell 124: 343-354.

Dunlap JC. 2006. Proteins in the Neurospora circadian clockworks. J Biol Chem 281: 28489-28493.

Freitag M, Hickey PC, Khlafallah TK, Read ND, Selker EU. 2004. HP1 is essential for DNA methylation in Neurospora. Mol Cell 13: 427-434.

Galagan JE, Selker EU. 2004. RIP: the evolutionary cost of genome defense. Trends Genet 20: 417-423.

Grewal SI. 2010. RNAi-dependent formation of heterochromatin and its diverse functions. Curr Opin Genet Dev 20: 134-141.

Guo J, Cheng P, Yuan H, Liu Y. 2009. The exosome regulates circadian gene expression in a posttranscriptional negative feedback loop. Cell 138: 1236-1246.

Halic M, Moazed D. 2010. Dicer-independent primal RNAs trigger RNAi and heterochromatin formation. Cell 140: 504-516.

Heintzen C, Liu Y. 2007. The Neurospora crassa circadian clock. Adv Genet 58: 25-66.

Hobson DJ, Wei W, Steinmetz LM, Svejstrup JQ. 2012. RNA polymerase II collision interrupts convergent transcription. Mol Cell 48: 365-374.

Honda S, Selker EU. 2008. Direct interaction between DNA methyltransferase DIM-2 and HP1 is required for DNA methylation in Neurospora crassa. Mol Cell Biol 28: 6044-6055.

Iida T, Kawaguchi R, Nakayama J. 2006. Conserved ribonuclease, Eril, negatively regulates heterochromatin assembly in fission yeast. Curr Biol 16: 1459-1464.

Kampa D. 2004. Novel RNAs identified from an in-depth analysis of the transcriptome of human chromosomes 21 and 22. Genome Res 14: 331-342.

Katayama S. 2005. Antisense transcription in the mammalian transcriptome. Science 309: 1564-1566.

Kennedy S, Wang D, Ruvkun G. 2004. A conserved siRNA-degrading RNase negatively regulates RNA interference in $C$. elegans. Nature 427: 645-649.

Kim J, Guermah M, Roeder RG. 2010. The human PAF1 complex acts in chromatin transcription elongation both independently and cooperatively with SII/TFIIS. Cell 140: 491-503.

Kowalik KM, Shimada Y, Flury V, Stadler MB, Batki J, Buhler M. 2015. The Paf1 complex represses small-RNA-mediated epigenetic gene silencing. Nature 520: 248-252.

Kramer C, Loros JJ, Dunlap JC, Crosthwaite SK. 2003. Role for antisense RNA in regulating circadian clock function in Neurospora crassa. Nature 421: 948-952.

Lee HC, Li L, Gu W, Xue Z, Crosthwaite SK, Pertsemlidis A, Lewis ZA, Freitag M, Selker EU, Mello CC, et al. 2010. Diverse pathways generate microRNA-like RNAs and Dicer- independent small interfering RNAs in fungi. Mol Cell 38: 803-814.

Lewis ZA, Adhvaryu KK, Honda S, Shiver AL, Knip M, Sack R, Selker EU. 2010. DNA methylation and normal chromosome behavior in Neurospora depend on five components of a histone methyltransferase complex, DCDC. PLOS Genet 6: e1001196.

Li H, Handsaker B, Wysoker A, Fennell T, Ruan J, Homer N, Marth G, Abecasis G, Durbin R, Proc GPD. 2009. The Sequence Alignment/Map format and SAMtools. Bioinformatics 25: 2078-2079.

Li Q, Su Z, Xu X, Liu G, Song X, Wang R, Sui X, Liu T, Chang X, Huang D. 2012. AS1DHRS4, a head-to-head natural antisense transcript, silences the DHRS4 gene cluster in cis and trans. Proc Natl Acad Sci 109: 14110-14115.

Li N, Joska TM, Ruesch CE, Coster SJ, Belden WJ. 2015. The frequency natural antisense transcript first promotes, then represses, frequency gene expression via facultative heterochromatin. P Natl Acad Sci 112: 4357-4362.

Magistri M, Faghihi MA, St Laurent G III, Wahlestedt C. 2012. Regulation of chromatin structure by long noncoding RNAs: focus on natural antisense transcripts. Trends Genet 28: 389-396.

Margolin BS, Garrett-Engele PW, Stevens JN, Fritz DY, GarrettEngele C, Metzenberg RL, Selker EU. 1998. A methylated Neurospora 5S rRNA pseudogene contains a transposable element inactivated by repeat-induced point mutation. Genetics 149: 1787-1797.

Nagano T, Mitchell JA, Sanz LA, Pauler FM, Ferguson-Smith AC, Feil R, Fraser P. 2008. The Air noncoding RNA epigenetically silences transcription by targeting G9a to chromatin. Science 322: $1717-1720$.

Navarro P, Page DR, Avner P, Rougeulle C. 2006. Tsix-mediated epigenetic switch of a CTCF-flanked region of the Xist promoter determines the Xist transcription program. Genes Dev 20: 2787-2792.

Niranjanakumari S, Lasda E, Brazas R, Garcia-Blanco MA. 2002. Reversible cross-linking combined with immunoprecipitation to study RNA-protein interactions in vivo. Methods 26: 182-190.

Osato N, Suzuki Y, Ikeo K, Gojobori T. 2007. Transcriptional interferences in cis natural antisense transcripts of humans and mice. Genetics 176: 1299-1306.

Pall GS, Hamilton AJ. 2008. Improved Northern blot method for enhanced detection of small RNA. Nat Protoc 3: 1077-1084.

Penheiter KL, Washburn TM, Porter SE, Hoffman MG, Jaehning JA. 2005. A posttranscriptional role for the yeast Paf1-RNA polymerase II complex is revealed by identification of primary targets. Mol Cell 20: 213-223.

Pomraning KR, Smith KM, Freitag M. 2009. Genome-wide high throughput analysis of DNA methylation in eukaryotes. Methods 47: 142-150.

Prescott EM, Proudfoot NJ. 2002. Transcriptional collision between convergent genes in budding yeast. Proc Natl Acad Sci 99: 8796-8801.

Quinlan AR, Hall IM. 2010. BEDTools: a flexible suite of utilities for comparing genomic features. Bioinformatics 26: 841-842.

Robinson JT, Thorvaldsdottir H, Winckler W, Guttman M, Lander ES, Getz G, Mesirov JP. 2011. Integrative genomics viewer. Nat Biotechnol 29: 24-26.

Rondón AG, Gallardo M, García-Rubio M, Aguilera A. 2004. Molecular evidence indicating that the yeast PAF complex 
is required for transcription elongation. EMBO Rep 5: 47-53.

Rountree MR, Selker EU. 2010. DNA methylation and the formation of heterochromatin in Neurospora crassa. Heredity (Edinb) 105: 38-44.

Sun BK, Deaton AM, Lee JT. 2006. A transient heterochromatic state in Xist preempts X inactivation choice without RNA stabilization. Mol Cell 21: 617-628.

Tomson BN, Arndt KM. 2013. The many roles of the conserved eukaryotic Pafl complex in regulating transcription, histone modifications, and disease states. Biochim Biophys Acta 1829: $116-126$.

Tufarelli C, Stanley JA, Garrick D, Sharpe JA, Ayyub H, Wood WG, Higgs DR. 2003. Transcription of antisense RNA leading to gene silencing and methylation as a novel cause of human genetic disease. Nat Genet 34: 157-165.

Volpe TA, Kidner C, Hall IM, Teng G, Grewal SI, Martienssen RA. 2002. Regulation of heterochromatic silencing and histone H3 lysine-9 methylation by RNAi. Science 297: $1833-1837$.

Wang T, Xie Y, Xiao GH. 2014. dCLIP: a computational approach for comparative CLIP-seq analyses. Genome Biol 15: R11.
Xi Q, Gao N, Zhang X, Zhang B, Ye W, Wu J, Zhang X. 2014. A natural antisense transcript regulates acetylcholinesterase gene expression via epigenetic modification in Hepatocellular Carcinoma. Int J Biochem Cell Biol 55: 242-251.

Xue ZH, Ye QH, Anson SR, Yang JC, Xiao G, Kowbel D, Glass NL, Crosthwaite SK, Liu Y. 2014. Transcriptional interference by antisense RNA is required for circadian clock function. $\mathrm{Na}$ ture 514: 650-+.

Yang XC, Purdy M, Marzluff WF, Dominski Z. 2006. Characterization of $3^{\prime} \mathrm{hExo}$, a $3^{\prime}$ exonuclease specifically interacting with the $3^{\prime}$ end of histone mRNA. I Biol Chem 281: 3044730454.

Yu W, Gius D, Onyango P, Muldoon-Jacobs K, Karp J, Feinberg AP, Cui H. 2008. Epigenetic silencing of tumour suppressor gene p15 by its antisense RNA. Nature 451: 202-206.

Zhao J, Sun BK, Erwin JA, Song JJ, Lee JT. 2008. Polycomb proteins targeted by a short repeat RNA to the mouse X chromosome. Science 322: 750-756.

Zhao Y, Shen Y, Yang S, Wang J, Hu Q, Wang Y, He Q. 2010. Ubiquitin ligase components Cullin4 and DDB1 are essential for DNA methylation in Neurospora crassa. I Biol Chem 285: 4355-4365. 


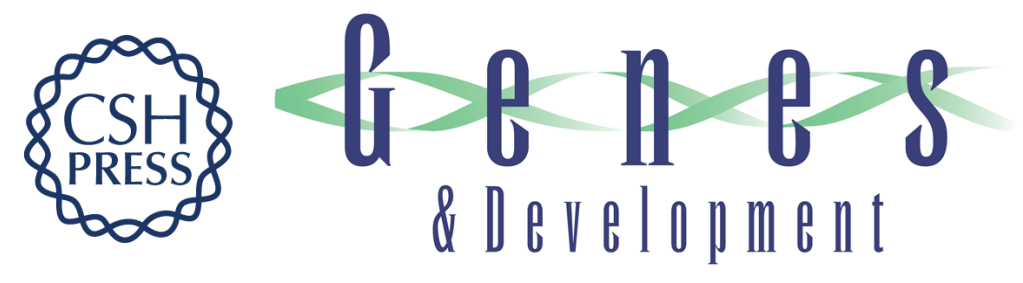

\section{Antisense transcription licenses nascent transcripts to mediate transcriptional gene silencing}

Yunkun Dang, Jiasen Cheng, Xianyun Sun, et al.

Genes Dev. 2016, 30: originally published online November 17, 2016

Access the most recent version at doi:10.1101/gad.285791.116

\section{Supplemental http://genesdev.cshlp.org/content/suppl/2016/11/17/gad.285791.116.DC1 Material}

References This article cites 66 articles, 23 of which can be accessed free at: http://genesdev.cshlp.org/content/30/21/2417.full.html\#ref-list-1

Creative This article is distributed exclusively by Cold Spring Harbor Laboratory Press for the first Commons six months after the full-issue publication date (see

License http://genesdev.cshlp.org/site/misc/terms.xhtml). After six months, it is available under a Creative Commons License (Attribution-NonCommercial 4.0 International), as described at http://creativecommons.org/licenses/by-nc/4.0/.

Email Alerting Receive free email alerts when new articles cite this article - sign up in the box at the top Service right corner of the article or click here.

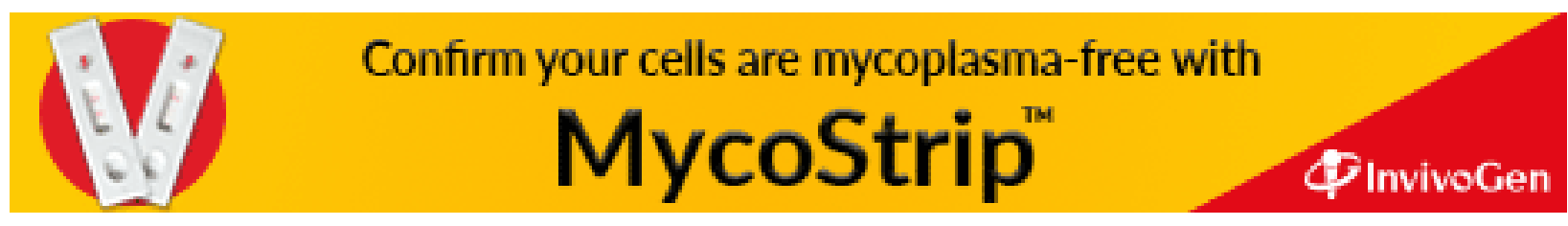

